\title{
Quantum Theory and Relativity
}

\author{
Arthur Jaffe* \\ Harvard University \\ Cambridge, MA 02138, USA \\ Arthur_Jaffe@harvard.edu
}

August 1, 2007

\section{Dedicated to George Mackey}

\begin{abstract}
Elsewhere I describe some personal encounters with George Mackey [31]. Those discussions often returned to a common set of questions:

- Does physics suggest a way to combine quantum theory, relativity, and interaction?

- To what extent can one formulate such quantum physics as mathematics?

- What is the present status of quantum field theory?

- What new mathematics emerges?

- What new insight does one apparently require to make further progress?

We touch on these topics here, not only outlining some of the technical issues, but also attempting to address why one believes in their importance.
\end{abstract}

\section{Introduction}

Two major themes dominated twentieth century physics: quantum theory and relativity. These two fundamental principles provide the cornerstones upon which one might build the understanding of modern physics. And today after one century of elaboration of the original discoveries by Poincaré, Einstein, Bohr, Schrödinger, Heisenberg, Dirac - and many others - one still dreams of describing the forces of nature within such an arena. Yet we do not know the answer to the basic question:

\section{- Are quantum theory, relativity, and interaction mathematically compatible?}

Even if one restricts relativity to special relativity, we do not know the answer to this question about our four-dimensional world - much less about other higher-dimensional worlds considered by string theorists!

Should quantum theory with relativity not qualify as logic? Physics suggests that a natural way to combine quantum theory, special relativity, and interaction is through a non-linear quantum field. Enormous progress on this problem has been made over the past forty years. This includes showing that theories exist in space-times of dimension two and three. Building this new mathematical framework and finding these examples has become known as the subject of constructive quantum field theory.

We review some of these developments here. But before addressing this question further, we set down a couple of ground rules. Although one cannot derive these starting points from basic first principles, we attempt to explain the road we take.

\footnotetext{
*I am grateful to the I.T.P. of the E.T.H. Zürich for hospitality while I wrote this paper, and to Barbara Drauschke for proof-reading the manuscript.
} 
Scope. First let us ask about the domain of validity for the "laws of physics." In searching for these laws, can one be content in finding laws that are effective within a limited domain of naturesuch as laws that describe atoms and molecules, or laws that pertain to strong interactions and electrodynamics, but that touch on neither gravity nor weak interactions? Or can one only be satisfied when one can formulate a theory that encompasses all of physics?

For example, special relativity and the notion of Minkowski space-time revolutionized the notion of classical physics. But one might argue that space-time itself cannot remain strictly Minkowskilike at extremely high scales of energy or in the neighborhood of a black hole; so should one only study theories where space-time can have a more general character-possibly curved, or possibly with a quantum nature, or both? Can one justify, from the point of view of physics, the study of special relativity in great detail? How much human thought and effort should one invest in giving a mathematical foundation to an incomplete picture of nature?

Our position on this question is that past success in understanding physics has always been partial and within limited domains. But within these domains one has pursued theories to their logical conclusion. Insight into broad arenas of physics followed studies that ranged from Newton's laws of planetary motion, to extremal principles in the laws of mechanics and optics, Maxwell's laws of classical electromagnetism, probabilistic laws of Brownian motion, statistical foundations of thermodynamics and phase transitions, and non-relativistic quantum theory - to mention just a few.

Yet each of these examples is specific to a certain domain of relevance. A big part of the success and the appeal of these "theories" rests with their elegance and simplicity, and another appeal is the depth of understanding they provide within each realm of physics. These theories not only model parts of nature; but in addition they yield lasting beacons on the frontiers of knowledge - markers to which one returns time and time again.

Of course one constantly strives to widen the scope of our understanding. As we acquire more knowledge, we expand our horizons, always requiring that new points of view incorporate previous successes and insights. Some physicists have proposed very ambitious plans; at one limit, string theorists try to find a "theory of everything." Laudable though this goal may be, the formulation of all-encompassing principles has remained elusive.

With this as background, we state our first ground rule: it is valid to address the partial goal, are quantum theory, special relativity and interaction compatible?

Logic. If one accepts an intermediate and partial goal in the description of nature, then one confronts a related question: why require that a partial theory have a firm foundation in logic? In other words, why provide a valid mathematical basis for a partial set of physical laws? Is it not better to focus effort on discovering new principles that describe a wider spectrum of natural phenomena? If one accepts that view, then one might only attempt to clarify the logical foundations of physics after achieving a much better, if still putative understanding of nature.

Our response to that proposal is another appeal to history. Each of the classical areas of physics mentioned under 'scope' qualifies as a subfield of mathematics. For centuries, the tradition in physics has been to describe natural phenomena by mathematics. Eugene Wigner marveled on the relevance of mathematics in his famous essay [60], "On the Unreasonable Effectiveness of Mathematics in the Natural Sciences." Intuition can go a long way. But by endowing physics with a mathematical foundation, one also bestows physical laws with longevity. For mathematical ideas can be understood and conveyed more easily than conjectures, both from person to person, and also from generation to generation.

In recent years we have witnessed enormous progress in another direction - of transferring ideas from physics to mathematics: to play on Wigner's title, concepts from physics have had an unreasonable effectiveness in providing insight to formulate mathematical conjectures! The resulting infusion of new perspectives has truly blossomed into a mathematical revolution, which has been sufficiently robust to touch almost every mathematical frontier. I have written and talked on this 
latter topic extensively elsewhere, but this is not the central theme that we investigate here.

Rather, we simply formulate the second ground rule: physics should be a subfield of mathematics! In other words, one should add the adjective mathematically to the word compatible in ground rule one. George Mackey often advocated this point of view in our conversations, and most every mathematician would find this an appealing thought. Being mathematics does not limit the use of insight or stop one making unjustifiable approximations to gain insight into a particular problemthe only requirement is to be clear about what parts of physics are mathematics and which parts are not.

The real question is whether this goal is realistic. No matter how laudable it may be to strive for a mathematical theory of physics, can one expect to achieve it? This brings us inevitably to the fundamental question posed at the outset. Understanding some self-contained, but complete nugget in this sea of knowledge can produce work of lasting historical value - a mathematical work of art.

Framework. We approach the question from the point of view of quantum field theory. In spite of tremendous qualitative progress mentioned above in understanding space-time dimensions less than four, the space-time in which we live still remains a mystery. So we first point out some facts that motivate hope for the success of this quest, namely the success of quantum electrodynamics. Then we explain some approaches to giving a mathematical foundation to quantum field theory. But we then return to explain the currently-widespread belief that a mathematical treatment of quantum electrodynamics may well be impossible!

If this were true, it would certainly represent a departure from traditional thinking - that one can study the electromagnetic forces by themselves. But today physicists believe that one should incorporate the equations of four-dimensional electrodynamics into the four-dimensional quantum Yang-Mills equations, or elaborate them into the "standard model."

In any case, the question of finding an interacting relativistic field in four dimensions appears even more subtle than one might at first believe. For this and other reasons, we believe that the question, "Does there exist a mathematically-complete, non-linear relativistic quantum field theory in Minkowski four-space?" remains one of the most important unresolved questions in all of science.

\section{Quantum Theory, Relativity, and Interaction}

Here is the picture: States in quantum theory lie in a Hilbert space $\mathcal{H}$. One assumes that there is a unitary representation $U$ of the Poincare group $G$ on $\mathcal{H}$. Single particle states are defined as subspaces $\mathcal{K} \subset \mathcal{H}$ that carry an irreducible representation of this group. There is a distinguished Poincaré-invariant state $\Omega \in \mathcal{H}$ which represents the physical "vacuum." A real quantum field $\varphi(x)$, when paired with a real, Schwartz-space function $f(x)$, yields a symmetric linear operator $\varphi(f)=\int \varphi(x) f(x) d x$ acting on $\mathcal{H}$. One says that $\varphi$ is an operator-valued distribution.

Single particle states arise from the states $\varphi(f) \Omega$. Multi-particle states occur in the application of several fields to the vacuum, and the forces between particles are a consequence of the nonlinearity of the field equation. In this way, one can derive the force law between particles directly from something fundamental - the form of the field equation. This non-linearity specifies the "laws of physics."

What other general features would one hope to incorporate? It is natural to expect that freelymoving particles emerge asymptotically at large times from states of the field describing particles moving away from one another. The fundamental properties of such states should arise from the behavior of $\varphi$ as a solution to a non-linear field equation. The particles would have fundamental properties as a consequence of the field equation, and this equation would also determine the interaction and scattering of an arbitrary number of particles.

For example, a cubic non-linear interaction $\frac{g}{3} \varphi^{3}$ for a scalar field with mass $m$, leads in lowest 
order perturbation theory to an attractive Yukawa-like force $V(r)=\frac{g^{2}}{4 \pi r} e^{-m r}$ between two particles. Basically the form of the force law in perturbation theory arises from the form of the Green's function for the time-independent linear equation. So one can ask whether this perturbation theory consequence, as well as many others, can be translated into properties of an actual non-linear theory?

\section{II.1 Compelling Evidence: Calculational Rules and Experiment}

One can reflect on the great initial triumphs of quantum field theory, the prediction and measurement of the Lamb shift in the spectrum of hydrogen and the value of the magnetic moment of the electron. These events which began in 1947 gave credence to the picture described above and heralded the era of the quantum field. They have been refined over sixty years to the point of unbelievable precision between experiment and computation.

The greatest quantitative triumph of this perturbation picture comes from the rules developed in quantum electrodynamics to calculate the magnetic moment of the electron. We focus on this, because it has evolved into one of the most accurate agreements between theoretical calculation and laboratory observation.

\section{II.2 Quantum Electrodynamics}

This year we celebrate the $60^{\text {th }}$-anniversary of the original calculation and observation of the deviation from its value that can be ascribed to the interaction between the electron (Dirac) field $\psi(x)$ and the electromagnetic (Maxwell) field $F(x)=(\vec{E}(x), \vec{B}(x))$. The heroic theoretical work of Bethe, Weisskopf, Feynman, Schwinger, Tomonaga, Karplus, Kroll, and others led to the original observation by Kusch in 1947.

Prior to 1947, there had been an evolution of understanding of this moment. A simple model of the electron as a current loop with angular momentum $J$ led to the picture that it would have a

magnetic moment $\mu=e J / 2 m c$. Taking the quantum value $J=\frac{1}{2} \hbar$ for the angular momentum, this meant the magnetic moment predicted by Bohr would be

$$
\mu_{\mathrm{Bohr}}=\frac{e \hbar}{4 m c} .
$$

In a magnetic field of magnitude $B$, the electron would have a potential energy $\mu B$, and this was the original prediction of non-relativistic quantum theory.

The Dirac equation for a single relativistic electron naturally incorporates both special relativity and the interaction with the magnetic field. It also predicts twice the interaction energy of the nonrelativistic theory that one could attribute to a magnetic moment of the electron. In other words, the Dirac value of the magnetic moment would be

$$
\mu_{\text {Dirac }}=\frac{e \hbar}{2 m c} .
$$

This was the value seen in observations.

However the proposal for a field theory explanation of the interaction between light and matter led to a further refinement. The natural assumption about the form of the interaction introduced the simplest non-linearity into the equations of quantum mechanics. The electron described by the Dirac equation and the classical theory of light developed in the previous century by Maxwell could be coupled similarly to the theory of classical electron motion in a magnetic field. One assumes that the non-linear Lagrangian is $\int J \cdot A d \vec{x}$, where the $A(x)$ denotes the Maxwell potential and where $J(x)=e \bar{\psi}(x) \gamma \psi(x)$ is the electric current. Here one takes $J(x)$ as the same quadratic function of the Dirac field $\psi(x)$ that appeared in the original Dirac theory. The matrices $\gamma$ are the famous 
$4 \times 4$ Dirac matrices; they satisfy the relation $\gamma^{\mu} \gamma^{\nu}+\gamma^{\nu} \gamma^{\mu}=2 g^{\mu \nu}$, similar to a Clifford algebra, but incorporating the Minkowski metric $g^{\mu \nu}$.

The problem with this procedure is that energy shifts due to the perturbation of the Lagrangian for a linear wave equation turned out to be infinite. Eventually physicists devised a set of rules to perform the calculations of observable effect. These rules involved taking infinite values of the mass and charge in the original equations, while requiring that the resulting perturbed masses and charges agree with observed values.

This non-linear field theory gave rise to rules of calculation in perturbation theory. One computed that the electromagnetic field interacted with and modified the magnetic moment of the electron. In 1947 Kusch, working at Columbia University, measured the change in the magnetic moment. It agreed precisely with the calculated effect that is first-order in the square of the electric charge, namely

$$
\mu_{\text {Kusch }}=\kappa \mu_{\text {Dirac }}, \quad \text { where } \kappa=1.001 .
$$

This tiny increase of only 0.1 percent compared with the Dirac value could be measured, and ever since one puts great credence in quantum field theory.

Over the intervening years, the measurements of $\mu$ and other related quantities has been refined by many persons, especially Dehmelt and his student Gabrielse. Likewise the calculations have undergone enormous progress. In order to take the accuracy to terms proportional to $e^{4}$, one required enormous computer power just to simplify the algebra required to multiply $4 \times 4$ matrices and calculate the relevant traces, much less compute the many integrals of rational functions (Feynman diagrams). A great expert who carried out much of the theoretical work is T. Kinoshita.

On this $60^{\text {th }}$-anniversary of the 1947 measurement, one can test the value of $\mu$ to unbelievable accuracy. The latest result, see [43, 15], cited by the American Institute of Physics as the "outstanding physics achievement of 2006," gives

$$
\mu=\kappa_{60} \mu_{\text {Dirac }}, \quad \text { where } \kappa_{60}=1.00115965218085( \pm 76) .
$$

The calculations and theory agree completely to this extent. And the accuracy of this test astounds the human mind. Thus while other physical theories (such as string theory or its ramifications) may ultimately predict new observed phenomena, any such theory must also reproduce the predictions of quantum electrodynamics including (II.4). Miraculously, these rules work in a precise quantitative fashion, indicating that they might reflect a well-behaved underlying theory that we do not yet understand.

\section{Sense or Nonsense?}

Faced in the early 1950's with the astounding successes of the field concept in physics, as well as the apparent robustness of the calculational rules, one could rejoice. However from a fundamental point of view, quantum fields baffled all insight; what could be the meaning of equations of physics that involve infinite constants and undefined operations? So we come back to the second question, "To what extent can one formulate relativistic quantum physics with interaction as mathematics?"

Mathematicians and physicists achieved a love-hate relationship with quantum field theory. Richard Feynman described renormalization, a subject that he and others pioneered, in terms of a magician's "hocus pocus," see page 128 of [9]. Yet he also expressed a keen interest in knowing whether relativistic quantum physics, and quantum electrodynamics in particular, has a mathematical foundation; see for example [8]. Many mathematicians held a distant view of quantum physics, and not only because its foundations were imprecisely laid out. Many mathematicians, especially those from the Bourbaki school, felt that mathematics itself needed to be put in order. They also regarded modern physics as beyond the scope of mathematical understanding. 
Faced with this gulf in knowledge, a number of mathematicians and physicists attempted in the 1950's to formulate a mathematical framework in which quantum field theory might fit. These efforts became known as Axiomatic QFT.

\section{III.1 Axiomatic Quantum Field Theory}

The early work, pioneered by Wightman, Jost, and Haag focused on formulating reasonable principles one could require for any quantum field. The outline of this framework can be found in [56] with elaboration in [57]. Full presentations and consequences of the axioms can be read in [53, 36].

One begins with the requirement that quantum theory satisfies some very basic properties which have become known as the Wightman axioms. Basically they embody the following requirements:

- States lie in a quantum-mechanical Hilbert space $\mathcal{H}$.

- There is a unitary, positive-energy representation $U$ of the Poincaré group on $\mathcal{H}$.

- There exists an invariant, vacuum-vector $\Omega=U \Omega \in \mathcal{H}$.

- The quantum field $\varphi$ is an operator-valued distribution.

- Vectors of the form $\varphi\left(f_{1}\right) \cdots \varphi\left(f_{n}\right) \Omega$, for $f \in \mathcal{S}$ and arbitrary $n$ span $\mathcal{H}$.

- The field $\varphi$ transforms covariantly under $U$.

- The field $\varphi$ is local.

- The space of invariant vectors $\Omega$ is one-dimensional.

Technically the Poincare group $G=\{\Lambda, a\}$ is the semidirect product of the Lorentz group (the group of $4 \times 4$ matrices $\Lambda$ that preserve the fundamental Minkowski quadratic form $t^{2}-\vec{x}^{2}$ ), with the abelian group $R^{4}$ of space-time translations $x \mapsto x+a$. The composition law for the group is $\left\{\Lambda_{1}, a_{1}\right\}\left\{\Lambda_{2}, a_{2}\right\}=\left\{\Lambda_{1} \Lambda_{2}, \Lambda_{1} a_{2}+a_{1}\right\}$. Aside from the identity representation, the irreducible, unitary representations of this group are infinite dimensional. On this account, one also requires strong continuity of the unitary representation.

The strongly-continuous, unitary, irreducible, positive-energy representations of the Poincaré group were dear to the heart of George Mackey. Many of these representations had been discovered by the physicist Majorana in 1932 [38], and they were also worked on by Dirac and Proca. The mathematical theory of the irreducible representations of the Poincaré group appeared in the groundbreaking 1939 paper of Eugene Wigner [59]. This work formed a motivating example for Mackey's theory of induced representations.

If one considers the connected components of the Poincaré group (by ignoring reflections), these representations are characterized in the case of space-time dimension $d=4$ by two parameters $\{m, s\}$ called mass and spin. The mass $m \geq 0$ is non-negative and the spin $s$ is half-integer. The representations of the group with reflections can be constructed from those of the group without reflections, and both sorts of representations occur in the description of different particles in physics.

The self-adjoint, infinitesimal generators of the representations of various subgroups of the Poincaré group play a special role in quantum physics. In particular, one writes the space-time translation subgroup $U(I, x)$ for $x=(\vec{x}, t)$ as

$$
U(x)=e^{i t H / \hbar-i \vec{x} \cdot \vec{P} / \hbar} .
$$

The self-adjoint operator $H$ that generates time-translation is called the Hamiltonian, and it is identified with the energy in physics. The self-adjoint generators $\vec{P}$ of spatial translations are called 
the momentum. For simplicity, for the remainder of this article we use units in which Planck's constant $\hbar=1$, and also the velocity of light $c=1$.

As the representation $U$ is infinite dimensional, one expects that the Hamiltonian $H$ is unbounded. A basic supposition of axiomatic field theory is that the energy has positive spectrum. (One says that $U$ is a positive energy representation.) Generally one adds a constant to adjust the infimum of the spectrum to zero. The mass operator $M$ is defined as the positive square root of

$$
M^{2}=H^{2}-\vec{P}^{2} .
$$

The mass operator $M$ commutes with the representation $U$. The vacuum $\Omega$ lies in the null space of $M$, and one says the vacuum is unique if the null space is one-dimensional. If $M$ has an eigenvalue $m>0$, the corresponding eigenspace is called a one-particle space in case $U$ acts irreducibly on this eigenspace.

In the case of a scalar field $\varphi(f)$ covariance means that the unitary representation $U(\Lambda, a)$ of the Poincaré group acts as

$$
U(\Lambda, a) \varphi(f) U(\Lambda, a)^{*}=\varphi(\{\Lambda, a\} f), \quad \text { where }(\{\Lambda, a\} f)(x)=f\left(\Lambda^{-1}(x-a)\right) .
$$

Furthermore for a massive, scalar field, the subspace $\varphi(f) \Omega \in \mathcal{H}$ should lie in the representation space of an irreducible representation with parameters $m>0$ and $s=0$, when the Fourier transform of $f$ is localized near a hyperboloid $E^{2}-\vec{p}^{2}=m^{2}$ in energy-momentum space, where $m$ is an isolated point in the spectrum of the mass operator $M$.

The property of locality is the least intuitive of the axiomatic assumptions; it is a technical way to embody the notion of relativistic causality. In quantum theory, one associates observable quantities with self-adjoint linear operators on $\mathcal{H}$ (operators, for short). One says that the two self-adjoint observables can be measured simultaneously if they can be simultaneously diagonalized, namely if they commute. One also uses the term independent to describe commuting observables.

In case $f \in C_{0}^{\infty}$ real , then the assumption of locality requires that $\varphi(f) \varphi(g)=\varphi(g) \varphi(f)$ when the supports of $f$ and $g$ cannot be connected by a light ray. One says that such supports are space-like separated. The locality condition for fermion fields $\psi(f)$ is somewhat more complicated, as these fields are not assumed to be directly observable; rather quadratic functions of fermions are assumed to be observables. This leads to a formulation of the locality criterion for fermion fields as the anti-commutativity of the fermion fields, $\psi(f) \psi(g)=-\psi(g) \psi(f)$, if the supports of $f$ and $g$ are space-like separated.

From these assumptions, one was able to conclude interesting consequences, both about the analyticity of the expectations of products of fields, and also about symmetries of such field theories. One of the best-known conclusions is a proof of a relation between the spin of a particle (integer or half-integer) and its statistics (Bose-Einstein or Fermi-Dirac) respectively. The result is that the anticommutation of fields at space-like separation is incompatible with an integer-spin representation for single particles, and likewise commutation of fields at space-like separation is incompatible with a half-integer-spin representation for single particles. Another well-known conclusion is that nature has a discrete symmetry called PCT, involving parity, charge conjugation, and time reversal.

Lehmann, Symanzik, Zimmermann, and Haag and Ruelle showed how to incorporate scattering theory of (massive) particles into the axiomatic framework. They assumed more structure, especially the existence of isolated, massive, one-particle states that one can obtain by applying a field to the vacuum $\Omega$. They then derived the existence of the scattering matrix for an arbitrary number of particles, as well as formulas to compute the scattering matrix elements from the vacuum expectation values of products of fields. Discussion and references can be found in [53, 36, 28, 20]. The additional axiom of Haag and Ruelle enables a clean mathematical treatment of scattering for particles of mass $m>0$ (but it does not apply to the case of massless particles). The axiom corresponding to the lightest particle having mass $m$ says: 
- The mass operator $M$ has isolated eigenvalues, 0 and $m>0$, and a spectral (mass)-gap ( $0, m)$.

Haag later introduced a slightly more general axiomatic approach, emphasizing the algebraic properties of the fields, see [28]. Rather than studying the representation $U$ of $G$ on $\mathcal{H}$, he studied the representation of the automorphism group that $U$ implements on the algebra of field operators $\mathcal{A}$. In place of the vacuum vector, he studied the state (expectation) that it engenders, namely a normalized, positive, linear functional on $\mathcal{A}$. Yet another algebraic approach was introduced by Borchers, see [3], focusing on the algebra of test functions $f$ with which one pairs the fields.

These axiom schemes all appear simple and natural. However in themselves they embody very few details about the physics; no laws of interaction are given! Thus one appears to have an enormous freedom in the choice of examples. But for many years after the original study of this framework in the 1960's, there were only two sorts of examples that satisfy any of the axiom schemes. And neither sets of these examples describe non-trivial interaction between particles, so they both are lacking from the point of view of physics.

The first set of examples are the free fields, which satisfy linear equations and describe freely moving (non-interacting) particles. The fields provided motivation for the formulation of the axioms. While from the point of view of physics, freely moving particles are not extremely interesting, they do provide a basis for discussion, and their existence shows that one can realize all the axiom schemes. Furthermore if a set of interacting particles move away from one another, one expects that they behave asymptotically at large times like free particles. Thus one expects that the free-particle structure appears as a limiting case in the scattering theory of a field describing interaction.

The second set of examples were some exactly-soluble interactions in two-dimensional space time. These exactly soluble models also did not lead to a robust description of particle interaction, with scattering matrix elements often independent of the energy.

\section{III.2 Examples with Interaction and Constructive QFT}

However, free fields do not realize the hope that that one can describe the interaction particles through an elementary equation of evolution for a relativistically covariant field. Motivated by the lack of an interesting realization of axiomatic quantum field theory, a great deal of interest focused on the question of whether specific non-linear quantum fields could be found. One attempted to construct solutions to elementary non-linear equations that do not appear to be exactly integrable or soluble in closed form.

Furthermore perturbation theory of the linear, free-field equation did not provide a good route to find solutions. There was proof in some cases, and evidence in other cases, that the perturbation expansions that one finds in all physics texts would not converge. And establishing a convergent re-summation of perturbation series required a priori properties of a solution that one did not know exists.

Clearly one must look for guidance from perturbation theory on how to approach the formulation of the non-linear equations, and how to cancel their divergences. But one must also develop a new, non-perturbative existence strategy, including a non-perturbative theory of renormalization. This extensive investigation came to be known as constructive quantum field theory.

\section{No Interaction is No Problem: Free Fields}

Before getting into the heart of how to treat interactions, let us first explore the structure of the free-scalar field theory. If one considers freely-moving particles (corresponding to classical motion without acceleration) then it is easy to find a Hilbert space $\mathcal{H}$, a field $\varphi$, and a representation $U$ satisfying all the axiom schemes. The states of $n$ particles arise from applying a polynomial of degree $n$ in the field to the vacuum state $\Omega$. This field satisfies a linear Klein-Gordon wave equation. 
We illustrate here the case $m=1, s=0$. In dimension $d=4$ the space spanned by $\varphi(f) \Omega$ is the representation space for a mass-1, spin-0 irreducible representation of the Poincaré group. A similar construction holds for any $d$.

Consider for example a single particle moving freely in Minkowski space-time $M^{d}$ with coordinates $x=(\vec{x}, t)$. In quantum theory one formulates the state $h$ of a particle (the wave function) as a function $h(\vec{x})$ of the coordinate $\vec{x} \in R^{d-1}$ at a given time. (We assume that space is $d-1$ dimensional, with $d-1=3$ the standard case.) Compatibility with special relativity means that the one-particle subspace $\mathcal{K}$ is the representation space of the spin-zero, mass-one representation of (the Poincaré group) $G$.

Time evolution of the single-particle state $h$ is given by the inverse $U(t)^{*}=e^{-i t H}$ of the oneparameter time-translation subgroup of $G$. This group is also called the Schrödinger group of quantum theory, and $h(t)=U(t)^{*} h$ defines a solution to the Schrödinger equation $i d h(t) / d t=H h(t)$, with initial data $h$.

\section{IV.1 One Scalar Particle}

We give here the details of the scalar case, so we take $\mathcal{K}$ to be the representation space of the scalar, mass one-particle. This is the Sobolev space $H_{-\frac{1}{2}}\left(R^{d}\right)$ with the inner product

$$
\langle h, h\rangle_{\mathcal{K}}=\langle h, h\rangle_{H_{-\frac{1}{2}}\left(R^{d-1}\right)}=\int \overline{\tilde{h}(\vec{p})} \tilde{h}(\vec{p}) \frac{d \vec{p}}{2 \omega(\vec{p})} .
$$

Here $\omega(\vec{p})=\left(\vec{p}^{2}+1\right)^{1 / 2}$, and $\tilde{h}=\mathfrak{F} h$ denotes the Fourier transform ${ }^{1}$ of $h$,

$$
(\mathfrak{F} h)(\vec{p})=\tilde{h}(\vec{p})=\frac{1}{(2 \pi)^{d-1}} \int h(\vec{x}) e^{i \vec{p} \cdot \vec{x}} d \vec{x} .
$$

Consider the space-time translation subgroup $U(x)$ of (III.1). The natural choices of $H$ and $\vec{P}$ in Fourier space are

$$
\mathfrak{F}^{-1} H \mathfrak{F}=\omega(\vec{p}) \quad \text { and } \mathfrak{F}^{-1} \vec{P} \mathfrak{F}=\vec{p},
$$

which entail

$$
H=\omega=\left(-\Delta_{d-1}+1\right)^{1 / 2}, \quad \text { and } \vec{P}=-i \vec{\nabla},
$$

where $\Delta_{d-1}$ denotes the Laplacian on $R^{d-1}$. Note

$$
\omega^{2}=\vec{P}^{2}+1 \text {. }
$$

Thus the inner product can be expressed as

$$
\langle h, h\rangle_{H_{-\frac{1}{2}}}=\left\langle h,(2 \omega)^{-1} h\right\rangle_{L^{2}} .
$$

Also the mass operator $M$ has eigenvalue 1 on $\mathcal{K}=H_{-\frac{1}{2}}$. For $h \in H_{-\frac{1}{2}}$, the function

$$
f(x)=U(\vec{x}, t)^{*} h,
$$

is an $H_{-\frac{1}{2}}\left(R^{d-1}\right)$-valued solution to the Klein-Gordon equation,

$$
(\square+1) f(x)=0, \quad \text { where } \square=\frac{\partial^{2}}{\partial t^{2}}-\Delta_{d-1},
$$

\footnotetext{
${ }^{1}$ Note that throughout this paper we use the symbol $\tilde{f}$ to denote the Fourier transform of $f$. One must be careful to distinguish this from the symbol $\hat{f}$, which we reserve for later use: in $\S$ VII we define the quantization $\hat{f}$ of $f$.
} 
with initial data,

$$
f(\vec{x}, 0)=U(\vec{x}, 0) h, \quad f_{t}(\vec{x}, 0)=-i U(\vec{x}, 0) H h .
$$

The function $f(x)$ also satisfies the first order Schrödinger equation

$$
i \frac{\partial f(\vec{x}, t)}{\partial t}=H f(\vec{x}, t)
$$

with initial data $h(\vec{x})$.

In the case of the $s=\frac{1}{2}$ representation, the wave function $h(x)$ is spinor-valued and satisfies a first order differential equation (such equations were originally introduced by Dirac, Weyl, and Pauli). In the case of spin $s=1$ the wave function $h(x)$ is vector valued, etc. We continue the exposition of the scalar case.

\section{IV.2 Fock Space of Many Particles}

One can combine several particles without interaction by taking tensor powers of the Hilbert spaces for the single particles. With identical particles, one uses a symmetric tensor product to combine integer spin particles, or particles with integer spin and half-integer spin. One combines two particles with half-integer spin using the anti-symmetric tensor product.

A simple way to describe an arbitrary number of non-interacting particles is by a Hilbert space $\mathcal{H}$ that is the direct sum of the $n$-particle spaces $\mathcal{F}_{n}$. Let $\mathcal{F}_{0}=\mathfrak{C}$, and $\mathcal{F}_{1}=\mathcal{K}$, while for scalar particles, the $n$-particle space is the symmetric tensor product

$$
\mathcal{F}_{n}=\underbrace{\mathcal{K} \otimes_{s} \mathcal{K} \otimes_{s} \cdots \otimes_{s} \mathcal{K}}_{n \text { factors }}=\mathcal{K}^{\otimes_{s} n}
$$

with the inner product normalized so that $h^{\otimes n}=h^{\otimes_{s} n}$ has norm $n !^{1 / 2}\|h\|_{H_{-\frac{1}{2}}}^{n}$. One takes $\mathcal{H}$ as the Fock space over $H_{-\frac{1}{2}}\left(R^{d-1}\right)$, which is the direct sum of $n$-particle spaces,

$$
\mathcal{H}=\mathcal{F}=\oplus_{n=0}^{\infty} \mathcal{F}_{n}
$$

The unitary $U(x)$ acts on each tensor factor $\mathcal{K}$ in $\mathcal{H}_{n}$, with $n \geq 1$, and as the identity on $\mathcal{H}_{0}$. One often denotes the function $1 \in \mathcal{F}_{0}$ by $\Omega$, and calls this no-particle state the vacuum in $\mathcal{H}$. Also the vacuum is translation invariant, $U(x) \Omega=\Omega$, so the state $\Omega$ has zero energy and momentum (as well as zero angular momentum, etc.).

\section{IV.3 Desired Properties for the Free Scalar Field}

One desires the free scalar quantum field $\varphi(x)$ to be an operator-valued distribution that also is a solution to the Klein-Gordon equation

$$
(\square+1) \varphi(x)=0 .
$$

With a real Schwartz-space function $f \in \mathcal{S}\left(R^{d}\right)$, one desires that $\varphi(f)=\int \varphi(x) f(x) d x$ is a symmetric operator on $\mathcal{F}$, transforming according to (III.3). The differential equation (IV.13) could also be written as $\varphi((\square+1) f)=0$.

The free field solution has the property that it is sufficient to pair $\varphi$ with a test function on $R^{d-1}$. Let $\delta_{t}$ denote the Dirac measure localized at time $t$. Then for $h \in H_{-\frac{1}{2}}\left(R^{d-1}\right)$, the operator $\varphi\left(h \otimes \delta_{t}\right)$ should be a continuous function of $t$ on a dense domain. By choosing more regular functions $h$, the field $\varphi\left(h \otimes \delta_{t}\right)$ should become differentiable in $t$. Let

$$
\varphi_{t}(h, t)=\frac{\partial \varphi(h, t)}{\partial t}=[i H, \varphi(h, t)]
$$


One requires that the free field initial data satisfy the canonical commutation relations. For example, for real functions $h, h^{\prime} \in H_{\frac{1}{2}}$,

$$
\left[\varphi_{t}(h, 0), \varphi\left(h^{\prime}, 0\right)\right]=-i\left\langle h, h^{\prime}\right\rangle_{L^{2}\left(R^{d-1}\right)}, \quad \text { and }\left[\varphi(h, 0), \varphi\left(h^{\prime}, 0\right)\right]=\left[\varphi_{t}(h, 0), \varphi_{t}\left(h^{\prime}, 0\right)\right]=0 \text {. }
$$

\section{IV.4 Realization of the Free Field}

It is well-known that this structure can be realized on Fock space, and we now illustrate this. Consider a real function $h \in H_{-\frac{1}{2}}\left(R^{d-1}\right)$. Define the field $\varphi(h, 0)$ on the domain $\mathcal{F}_{n}$ by

$$
\varphi(h, 0)\left(h_{1} \otimes_{s} \cdots \otimes_{s} h_{n}\right)=h \otimes_{s} h_{1} \otimes_{s} \cdots \otimes_{s} h_{n}+h \vdash\left(h_{1} \otimes_{s} \cdots \otimes_{s} h_{n}\right) .
$$

The first term $h \otimes_{s}$ of multiplication in the symmetric tensor product is sometimes called the creation operator $A(h)^{*}$ as one can interpret it mapping $\mathcal{F}_{n}$ to $\mathcal{F}_{n+1}$ as creating a particle. The second term in (IV.16) is the adjoint $h \vdash$ of $(h \otimes)^{*}$, and is sometimes called the annihilation operator $A(h)$. It is the contraction $h \vdash: \mathcal{F}_{n} \mapsto \mathcal{F}_{n-1}$. Also $h \vdash 1=0$ on $\mathcal{F}_{0}$. In particular, the normalization of the inner product on $\mathcal{F}_{n}$ yields

$$
\varphi(h, 0)\left(h_{1} \otimes_{s} \cdots \otimes_{s} h_{n}\right)=h \otimes_{s} h_{1} \otimes_{s} \cdots \otimes_{s} h_{n}+\sum_{j=1}^{n}\left\langle h, h_{j}\right\rangle_{H_{-\frac{1}{2}}} h_{1} \otimes_{s} \cdots \not h_{j} \cdots \otimes_{s} h_{n} .
$$

Here $h_{j}$ denotes the omission of the term $h_{j}$ from the product. This defines the time-zero field $\varphi(h, 0)$ as a symmetric operator on $\mathcal{F}$. In the case $n=0$, the second term in (IV.17) is absent and the range of the field is the one-particle space $H_{-\frac{1}{2}}$. In other words,

$$
\varphi(h, 0) \Omega=h .
$$

Thus the relation between the free field and its particle interpretation is built into this definition.

The Poincaré transformation group $U(g)$ action on the field $U(g) \varphi(h, 0) U(g)^{*}$ follows as a consequence of the action of $U(g)$ on the one-particle space, and its action as a tensor product on $\mathcal{F}_{n}$. Thus it follows from (IV.17) that

$$
U(g) \varphi(h, 0) U(g)^{*}=U(g) h \otimes_{s}+U(g) h \vdash .
$$

In the case of the time translation $U(t)$, one defines

$$
\varphi(h, t)=U(t) \varphi(h, 0) U(t)^{*}=e^{i t \omega} h \otimes_{s}+e^{i t \omega} h \vdash .
$$

One obtains the space-time averaged field $\varphi(f)$ for real $f \in \mathcal{S}\left(R^{d}\right)$ by integrating the field

$$
\left.\varphi\left(h^{(t)}, t\right)\right), \quad \text { where } h^{(t)}(\vec{x})=f(\vec{x}, t),
$$

over the time $t$. The action of $U$ on the one-particle space induces the covariant transformation law (III.3) for the field $\varphi(f)$. It is also easy to verify that $\varphi(f)$ is essentially self-adjoint on the domain of vectors with a finite number of particles. From this definition of $\varphi(f)$, one can derive the other desired properties of the free field: the canonical commutation relations, the fact that $\varphi$ satisfies the Klein-Gordon equation, and locality.

For example, for $h \in H_{\frac{1}{2}}$ one can differentiate $\varphi(h, t)$ in (IV.20) with respect to $t$ on the domain of states with a finite number of particles to obtain the time derivative $\pi(h, t)$ given by

$$
\pi(h, t)=\varphi_{t}(h, t)=i e^{i t \omega} \omega h \otimes_{s}-i e^{i t \omega} \omega h \vdash .
$$


Also for $h \in H_{3 / 2}$ we obtain the second time derivative,

$$
\varphi_{t t}(h, t)=-\varphi\left(\omega^{2} h, t\right)=-\varphi\left(\left(-\Delta_{d-1}+1\right) h, t\right) .
$$

This shows that the space-time field $\varphi(f)$ satisfies the Klein-Gordon equation (IV.13).

One can also verify the canonical commutation relations for the time-zero fields using (IV.22) and the definition (IV.17). One finds,

$$
\left[\pi(h, 0), \varphi\left(h^{\prime}, 0\right)\right]=-2 i\left\langle\omega h, h^{\prime}\right\rangle_{H_{-\frac{1}{2}}\left(R^{d-1}\right)}=-i\left\langle h, h^{\prime}\right\rangle_{L^{2}\left(R^{d-1}\right)},
$$

as anticipated in (IV.15). Furthermore, as similar computation shows that the time-zero fields $\varphi(h, 0)$ and their conjugates all commute, $\left[\varphi(h, 0), \varphi\left(h^{\prime}, 0\right)\right]=\left[\pi(h, 0), \pi\left(h^{\prime}, 0\right)\right]=0$. As a consequence of this property and Poincaré covariance, the free field is local. In fact it satisfies all the axiom schemes mentioned above.

\section{Constructive Quantum Field Theory}

The first attempts emphasized getting a solution to some non-linear quantum field equation, compatible with the canonical constraint of quantum theory on the initial data. For example, one might search for the simplest example, the solution to the $\varphi^{4}$ equation,

$$
(\square+1) \varphi(x)+4 \lambda \varphi^{3}(x)=0,
$$

with $\lambda>0$ a parameter, and subject to the same constraint (IV.15) as in the free case.

The basic difficulty arises from the necessity for renormalization described above. The Hamiltonian operators introduced in physics did not appear to fit within any known mathematical settings. While axiomatic field theory provided a general goal, it did not appear to include the mathematical tools required to find specific examples. In one-dimensional, non-relativistic quantum theory one had learned to deal with singular Hamiltonians (such as $H=-\frac{d^{2}}{d x^{2}}+\delta(\vec{x})$, where the potential is not a multiplication operator, but is a Dirac measure). But quantum field Hamiltonians are much more singular than such familiar territory in functional analysis; they require more insightful methods than those common at the time.

In order to study the problems, one needs to introduce a mollification, or cut-off; solve the approximate problem; and pass to a limit of removing the cut-off. One should mention K. Friedrichs for his early conceptual insights [10] into questions of the shift in domains of the Hamiltonian due to renormalization. Some further investigation focused on the mollified problem [29, 52]. For some time one pursued various avenues to achieve the limiting theory, but this problem remained open for over ten years after the axiomatic framework had been formulated.

The Hamiltonian for the equation (V.1) without cutoffs on a two-dimensional cylinder was known to be densely defined, after normal ordering - an elementary renormalization described below. This sort of renormalization is required and is sufficient in two space-time dimensions. A breakthrough came in 1965 when E. Nelson showed that this Hamiltonian defined on a spatial circle is bounded from below [40]. Another major step occurred three years later when that Hamiltonian, as well as related Hamiltonians, were proved to be essentially self-adjoint [17]. The self-adjointness enabled one to establish the existence of a finite-volume field theory, and it opened an era of rapid-fire progress with the solution of a succession of apparently difficult problems.

In another five years one had in hand the existence of the infinite volume limit of this field theory (namely a covariant non-linear field, as well as a unitary representation of the Poincaré group acting on a Hilbert space $\mathcal{H}$ with a unique invariant vector $\Omega$. For small $\lambda>0$, one knows that the spectrum of the Hamiltonian $H$, restricted to the subspace of zero momentum, has an isolated 
eigenvalue at 0 and a spectral mass gap $(0, m)$. Furthermore, restricted to the subspace of zero momentum $P$ (which commutes with $H$ ) the Hamiltonian has a spectral upper gap $(m, 2 m)$. It also has continuous spectrum in the interval $[2 m, \infty]$ see [22]. Analogous results were also proved for a family of related equations on two-dimensional Minkowski space. While it is known that the equation (V.1) leads to a repulsive interaction, more general equations may have a smaller upper gap $(m, 2 m-\epsilon)$ in case of the existence of bound states.

This work provided the first mathematically complete examples of interesting non-linear quantum fields. Not only do these fields satisfy all the versions of the axiom schemes mentioned above, but the perturbation theory of the mass eigenvalues or of the scattering matrix elements agrees with the perturbation theory that one finds in standard physics texts on quantum field theory. One can find an overview of this work in [30], and a self-contained presentation in [20].

\section{Two Methods}

Two basic methods arose in the investigation of constructive quantum field theory: the investigation of operator Hamiltonians $H$ that one obtained by canonical quantization, and the study of functional integration representations of matrix elements $\left\langle f, e^{-t H} g\right\rangle_{\mathcal{H}}$ of the heat kernel for $H$.

\section{VI.1 Canonical Hamiltonians}

The earliest proposals of Wightman, Jost, and Segal led to investigation of approximate Hamiltonians $H_{n}=H_{0}+H_{I, n}$ obtained through canonical quantization of systems with a finite number of degrees of freedom. Often one studied a perturbation $H_{n}$ of the free Hamiltonian $H_{0}$ (the Hamiltonian for the linear equation (V.1) with $\lambda=0$ ), as the free Hamiltonian $H_{0}$ can be defined without difficulty and without approximation. The interaction $H_{I, n}$ is generally expressed as the integral of an energy density, and the approximation $n$ indicates a $C_{0}^{\infty}$ coupling constant $0 \leq \lambda(\vec{x})$ and an ultraviolet mollification of the Fourier transform of $\varphi$. The interaction density is determined by a "potential" function $V$, which for the $\varphi^{4}$ theory is $V(\xi)=\xi^{4} / 4$, and where

$$
H_{I, n}=\int \lambda(\vec{x}) V\left(\varphi_{n}(\vec{x})\right) d \vec{x} .
$$

The Feynman-Kac formula allows one to give a classical interpretation to certain matrix elements of the heat kernel $e^{-t H}$, when $\varphi_{n}$ contains a finite number of Fourier modes. In the Schrödinger representation of the Hilbert space $\mathcal{H}$ for the free field, one represents the vector $f \in \mathcal{H}$ as an $L^{2}$ function of $\varphi(0)$ with respect to a measure on a space of paths $d \nu(\varphi(\cdot))$ on paths

$$
\left\langle f, e^{-t H} g\right\rangle_{\mathcal{H}}=\int_{\mathcal{S}^{\prime}} \overline{f(\varphi(0))} g(\varphi(t)) e^{-\int_{0}^{t} H_{I, n}\left(\varphi_{n}(s)\right) d s} d \nu(\varphi(\cdot)) .
$$

\section{VI.2 Euclidean Invariant Functional Integration in Physics}

Poincaré symmetry arises from the preservation of the Minkowski quadratic form $t^{2}-\vec{x}^{2}$. Analytic continuation of time from $t$ to purely imaginary time it yields the quadratic form $-x^{2}=-\sum_{i=1}^{d} x_{i}^{2}$, invariant under Euclidean transformations. The discovery by Schwinger of Euclidean field theory as an analytic continuation of Minkowski field theory began a long and fruitful investigation [49]. Schwinger also realized the importance of solving the inverse problem: how could one obtain a Poincaré-invariant theory from a Euclidean-invariant one? See the final paragraph on page 140 of $[50]$.

Let $T(O, a)$ denote the unitary representation on $\mathcal{E}$ of the Euclidean group $\{O, a\}$, namely the semi-direct product of rotations $O$ about the origin in $R^{d}$ and translations $a$. Euclidean scalar 
symmetry acts on elements $f \in \mathcal{S}\left(R^{d}\right)$ by

$$
(T(O, a) f)(x)=f\left(O^{-1}(x-a)\right) .
$$

In the Euclidean-invariant scalar theory, Jost showed that the analytically-continued time-ordered expectations are symmetric functions of the space-time points. In fact, in the Euclidean region one encounters classical fields in place of quantum fields!

The insightful physicist Kurt Symanzik advocated a variation of the Feynman-Kac method, namely the study of a classical Markov field in a Euclidean-invariant setting [54, 55]. Symanzik's papers are filled with information and difficult to digest. As a consequence, it took time to appreciate the overall beauty of Symanzik's ideas, many of which in hindsight appear simple and compelling, and which shape much of our modern way of thinking.

For the rest of this subsection, let us explain this program at the level of physics. Basically one attempts to define the statistical mechanics of a classical field in a Euclidean-invariant fashion. One attempts to attribute a Boltzmann-Gibbs statistical distribution to the configurations of the classical field of the form

$$
d \mu(\Phi)=\frac{1}{Z} e^{-\mathfrak{A}} d \Phi .
$$

Here $\mathfrak{A}$ denotes a Euclidean-invariant action functional of the classical field (which we denote here by $\Phi$ ), while $Z$ denotes the partition function or normalizing factor. The symbol $d \Phi$ indicates an average over field configurations. In fact, none of the three factors in the formula (VI.4) has a mathematical meaning. But one can try to ascribe a meaning to the product.

For the free wave equation (IV.13), one would choose $\mathfrak{A}$ to be the sum of a kinetic and potential term, both quadratic on the field,

$$
\mathfrak{A}=\mathfrak{A}_{0}=\frac{1}{2}\langle\nabla \Phi, \nabla \Phi\rangle_{L^{2}\left(R^{d}\right)}+\frac{1}{2}\langle\Phi, \Phi\rangle_{L^{2}\left(R^{d}\right)}=\frac{1}{2}\langle\Phi,(-\Delta+1) \Phi\rangle_{L^{2}\left(R^{d}\right)} .
$$

Here one ignores boundary terms.

In this Gaussian case, the answer is elementary. In this case one defines the product (VI.4) to be the well-defined and countably-additive Gaussian probability measure $d \mu_{C}(\Phi)$ on $\mathcal{S}^{\prime}\left(R^{d}\right)$ with mean zero and covariance $C=(-\Delta+1)^{-1}$.

On the other hand, if one studies the " $\varphi$ " equation (V.1), then one would choose

$$
\mathfrak{A}=\frac{1}{2}\langle\Phi,(-\Delta+1) \Phi\rangle_{L^{2}\left(R^{d}\right)}+\lambda\left\langle\Phi^{2}, \Phi^{2}\right\rangle_{L^{2}\left(R^{d}\right)}=\mathfrak{A}_{0}+\mathfrak{A}_{I} .
$$

One could attempt to understand the non-Gaussian (VI.4) as a perturbation of the Gaussian,

$$
d \mu(\Phi)=\frac{1}{Z} e^{-\lambda\left\langle\Phi^{2}, \Phi^{2}\right\rangle_{L^{2}\left(R^{d}\right)}} d \mu_{C}(\Phi),
$$

by studying an expansion about $\lambda=0$. One could study the distribution $d \mu(\Phi)$ either directly, or one can study its putative Fourier transform

$$
S(f)=\int e^{i \Phi(f)} d \mu(\Phi)
$$

or one can study its putative moments,

$$
S_{n}\left(f_{1}, \ldots, f_{n}\right)=\int \Phi\left(f_{1}\right) \cdots \Phi\left(f_{n}\right) d \mu(\Phi) .
$$

In the physics literature these moments are called Euclidean Green's functions, or Schwinger functions. 
The point of this approach is that instead of considering the Feynman-Kac formula describing diffusion in time $t$ of a field $\phi(\vec{x} ; t)$ taking values in a space of functions of $\vec{x}$, one regards diffusion in space-time of the field $\Phi(x)$. Thus $x$ plays the rôle of a "multi-dimensional time." This approach has many advantages, not the least of which is the explicit choice of a Euclidean-invariant form for the action $\mathfrak{A}$, so that one has an apparently Euclidean-invariant distribution $d \mu(\Phi)$ and Euclidean covariant moments.

However, this approach also came with a couple of apparent disadvantages:

- The study of Euclidean-invariant diffusion in multi-dimensional time was virgin mathematical territory, and it appeared mathematically intractable. (See the appendix by Varadhan to Symanzik's lectures [55].)

- The $\left|x-x^{\prime}\right|^{-(d-2)}$ singularity of the resolvent of the Laplacian on the diagonal means that generic paths in multi-dimensional time are not Hölder continuous, so the higher-degree terms in $\mathfrak{A}$ appear to be more singular.

Furthermore a debate emerged among the experts asking "is the canonical method or the functional integral method more insightful and robust?" Even Symanzik himself became caught up in this discussion, and sadly came to the conclusion that he should abandon his Euclidean method and also his study of constructive quantum field theory. It appeared at the time too difficult to develop the mathematical infra-structure necessary to implement this point of view, while considerable progress was being made through the study of canonical Hamiltonians, see [55].

Luckily within a short time it became clear that such debate was misdirected. The Hamiltonian and Euclidean methods complement one another, and the best results were obtained by combining them both. In fact, the understanding that the disadvantages above are really only "apparent" disadvantages, led to progress. One discovered a new class of mathematical objects - non-Gaussian, Euclidean-invariant, reflection-positive measures on $\mathcal{S}^{\prime}\left(R^{2}\right)$ and $\mathcal{S}^{\prime}\left(R^{3}\right)$. As a byproduct, this construction also led to the first mathematically complete examples of quantum field theories.

\section{The Euclidean Mathematical Framework}

The appeal of Symanzik's Euclidean field picture soon became evident, but it also raised the important puzzle alluded to in §VI.2. Suppose that one were able to construct a classical Euclideaninvariant field $\Phi$ with a non-Gaussian distribution $d \mu(\Phi)$. Then can one conclude that a corresponding Lorentz-invariant quantum field theory exists, and that this field acts on a Hilbert space $\mathcal{H}$ carrying an appropriate representation of the Poincaré group, and containing an invariant vacuum state $\Omega$ ? Or does one need extra assumptions in order to obtain a local quantum theory from studying Euclidean-invariant diffusion in multi-dimensional time?

Nelson gave the first mathematical description of Symanzik's Markov field in the Gaussian case [41], namely the free Euclidean Markov field $\Phi(x)$. This field is analogous to a $d$-dimensional timezero field, but with the covariance $C_{d}\left(x-x^{\prime}\right)$ replacing $C_{d}\left(\vec{x}-\vec{x}^{\prime}, 0\right)$. It provides a useful tool, and maps under the quantization described below onto the free quantum field $\varphi(x)$.

Nelson also provided a framework for how to get from a classical Euclidean-Markov field back to quantum theory [42]. The problem with this method is that it requires establishing a global Markov property. This property turns out to be difficult to verify - except in the free-field case. In fact it has not yet been established in most of the known examples of non-linear field theory. For this reason, and as the analog construction for fermion fields or other fields is not known, one hoped to find a more robust method.

Shortly afterward Osterwalder and Schrader, then young researchers at Harvard, discovered such a method. Their extremely simple idea reflection positivity (or RP) provides the key. It gives 
the answer for scalar fields, and it also applies in various forms to fields covariant under other representations. Checking RP in a free theory is not difficult, and for interacting theories (with well-chosen cutoffs) the proof of reflection positivity reduces to this property in the free-field case.

$\mathrm{RP}$ is also equivalent to certain classical monotonicity properties of Green's functions for the Laplacian with respect to introducing Dirichlet or Neumann data on hyper-surfaces. In the form used by Osterwalder and Schrader, this positivity condition has widespread application outside the context of relativistic quantum theory. It provides a framework to establish fundamental inequalities; it provides insights into the transfer matrix of statistical physics; and it has application in proving the analytic continuation of group representations. In fact, reflection positivity has roots in the much earlier work of Widder on analytic continuation of the Laplace transform [58].

\section{VII.1 Osterwalder-Schrader Axioms}

In order to simplify the following discussion, we restrict attention to scalar quantum fields. The Euclidean axioms appear simpler than the Wightman axioms of §III.1. They only entail

- A regularity assumption.

- Euclidean covariance.

- Reflection positivity.

- Clustering.

Osterwalder and Schrader formulated these axioms in terms of the Euclidean Schwinger functions, or Green's functions for a Euclidean field. They proved a remarkable equivalence theorem relating their axioms to the Wightman axioms of §III.1, see [46]. In particular, the property of locality is embodied in reflection positivity combined with Euclidean covariance!

Theorem VII.1 (Osterwalder and Schrader). The Osterwalder-Scharder axioms on Euclidean Green's functions (with a natural bound on the growth of the $n^{\text {th }}$ Green's functions) are equivalent to the Wightman axioms on the vacuum expectation values arising from the OS-quantization of these Green's functions, along with a growth condition on the $n^{\text {th }}$-vacuum expectation value. Omitting the clustering axiom of $O S$ is equivalent to omitting the unique-vacuum axiom of Wightman.

When the Euclidean expectation can be given by a functional integral $d \mu$, these axioms can be stated in terms of the Fourier transformation $S(f)=\int e^{i \Phi(f)} d \mu(\Phi)$ of the measure $d \mu$, see Fröhlich [11]. One says the the measure $d \mu(\Phi)$ is Euclidean-invariant if $S(T(O, a) f)=S(f)$ for all Euclidean transformations $T$ defined in (VI.3) and all real $f \in \mathcal{S}\left(R^{d}\right)$. Furthermore reflection positivity translates to the property that $S(f)$ is a function of reflection-positive type: for every choice of $n$ real functions $f_{j} \in \mathcal{S}\left(R_{+}^{d}\right)$ and complex constants $c_{j}$,

$$
0 \leq \sum_{i, j=1}^{n} \overline{c_{i}} c_{j} S\left(f_{j}-\Theta f_{i}\right)
$$

One also says that $S(f)$ is "regular," if the moments $\int \Phi(f)^{n} d \mu(\Phi)$ satisfy the growth of the Green's functions required by Theorem VII.1. One says that $S(f)$ clusters with an exponential rate $m$, if for all real $f, g \in C_{0}^{\infty}\left(R^{d}\right)$,

$$
|S(f+T(t) g)-S(f) S(g)| \leq O(1) e^{-m t} .
$$

Here the constant $O(1)$ may depend on $f, g$, and $T(t)$ denotes the time-translation subgroup $T(I,(\overrightarrow{0}, t))$. One arrives at a very interesting probabilistic consequence of this formulation. 
QuANTUm Theory and Relativity

Corollary VII.2. A Euclidean invariant, reflection-positive, regular probability measure $d \mu(\Phi)$ on $\mathcal{S}^{\prime}\left(R^{d}\right)$ yields a scalar quantum field theory satisfying the Wightman axioms on Minkowski space-time $M^{d}$. Clustering with exponential rate $m$ yields a mass gap $(0, m)$ in the spectrum of $H$.

For the remainder of this section, we explain the details of the formulation of the free Euclidean field in terms of these axioms, and how they yield a theory of free quantum fields.

\section{VII.2 "Euclidean" Test Functions}

The natural Sobolev space of test functions for the Euclidean field is $H_{-1}\left(R^{d}\right)$. Let $\Delta_{d}$ denote the Laplacian on $R^{d}$, and consider the standard Green's operator,

$$
0 \leq C=(-\Delta+1)^{-1} \text {. }
$$

Then $H_{-1}\left(R^{d}\right)$ is the Hilbert space with the inner product

$$
\langle f, g\rangle_{H_{-1}\left(R^{d}\right)}=\left\langle f,\left(-\Delta_{d}+1\right)^{-1} g\right\rangle_{L^{2}\left(R^{d}\right)}=\langle f, C g\rangle_{L^{2}\left(R^{d}\right)}=\left\langle\tilde{f}, \frac{1}{p^{2}+1} \tilde{g}\right\rangle_{L^{2}\left(R^{d}\right)} .
$$

In the last equality we use the unitary Fourier transform

$$
\tilde{f}(p)=\frac{1}{(2 \pi)^{d / 2}} \int e^{i p x} f(x) d x .
$$

Also single out one coordinate direction as "time", denoting a point in $R^{d}$ as $x=(\vec{x}, t)$. Denote the corresponding Fourier coordinate as $p=(\vec{p}, E)$. Introduce the operator $\omega=\left(-\Delta_{d-1}+1\right)^{1 / 2}$ on $L^{2}\left(R^{d-1}\right)$, which in Fourier space acts as multiplication by the function $\omega(\vec{p})=\left(\vec{p}^{2}+1\right)^{1 / 2}$. As $p^{2}+1=E^{2}+\omega(\vec{p})^{2}$, the Sobolev inner product has the representation

$$
\langle f, g\rangle_{H_{-1}\left(R^{d}\right)}=\int \frac{1}{E^{2}+\omega(\vec{p})^{2}} \overline{\tilde{f}(p)} \tilde{g}(p) d p .
$$

The Sobolev space $H_{-1}(X)$ on a subset $X \subset R^{d}$ is the Hilbert space obtained by completing the subspace of $L^{2}\left(R^{d}\right)$ functions that are supported in the set $X$ in the Sobolev norm determined by the inner product (VII.4). We often wish our space-time to be a union of a positive and negative time subspace, intersecting in a time-zero space:

$$
X=X_{+} \cup X_{-}, \quad \text { where } X_{+} \cap X_{-}=X_{0},
$$

where $X_{ \pm}$are interchanged by time reflection, and where $X_{0}$ is time reflection invariant. A common case we consider below is $X_{ \pm}=R_{ \pm}^{d}$, where $R_{+}^{d}$ is the positive-time half-space.

\section{VII.3 Reflection Positivity and Quantization}

Let $\Theta$ denote a unitary on $\mathcal{E}$ that we interpret as time reflection. Let $\mathcal{E}_{+}$denote the positive-time subspace of $\mathcal{E}$, and assume $\Theta \mathcal{E}_{ \pm}=\mathcal{E}_{\mp}$. Let

$$
\langle\cdot, \cdot\rangle_{\mathcal{H}}=\langle\Theta \cdot, \cdot\rangle_{\mathcal{E}} .
$$

Definition VII.3 (RP). If $0 \leq\langle f, f\rangle_{\mathcal{H}}$ for all $f \in \mathcal{E}_{+}$, we say that $\langle\cdot, \cdot\rangle_{\mathcal{H}}$ is reflection positive . In other words the time reflection $\Theta$ is a positive operator on $\mathcal{E}$ restricted to the domain $\mathcal{E}_{+}$,

$$
0 \leq \Theta \uparrow_{\mathcal{E}_{+}}
$$


Let $\mathcal{N}$ denote the null space of the form (VII.8) and complete the pre-Hilbert space $\mathcal{E}_{+} / \mathcal{N}$ in the norm given by (VII.8) to obtain the Hilbert space $\mathcal{H}$. We denote by hat ${ }^{\wedge}$ the quantization map for vectors. It is the identification map from $\mathcal{E}_{+}$to its equivalence class in $\mathcal{H}$. If (VII.8) is reflection positive, then ${ }^{\wedge}$ is a contraction, for

$$
\langle\hat{f}, \hat{f}\rangle_{\mathcal{H}}=\langle\Theta f, f\rangle_{\mathcal{E}} \leq\|f\|_{\mathcal{E}}^{2} .
$$

This means $\|\wedge\|_{\mathcal{E}_{+} \rightarrow \mathcal{H}} \leq 1$. We now explore a couple of examples with RP.

\section{VII.4 RP-1: A Single Free Particle, Revisited}

For the first example, take $\mathcal{E}=H_{-1}\left(R^{d}\right)$, and define the time reflection as $\Theta:(\vec{x}, t) \mapsto(\vec{x},-t)$. This $\Theta$ commutes with $\Delta_{d}$, and therefore lifts to a unitary on $H_{-1}\left(R^{d}\right)$. Denote the positive and negative half-spaces as $R_{ \pm}^{d}=R^{d-1} \times R_{ \pm}$, so time reflection exchanges these spaces, $\Theta: R_{ \pm}^{d}=R_{\mp}^{d}$, and also $\Theta: H_{-1}\left(R_{ \pm}^{d}\right)=H_{-1}\left(R_{\mp}^{d}\right)$. In this example $\mathcal{E}_{ \pm}=H_{-1}\left(R_{ \pm}^{d}\right)$.

Define the sesqui-linear form $\langle\cdot, \cdot\rangle_{\mathcal{H}}$ on $\mathcal{E}_{+} \times \mathcal{E}_{+}$by

$$
\langle\hat{f}, \hat{g}\rangle_{\mathcal{H}}=\langle\Theta f, g\rangle_{H_{-1}\left(R^{d}\right)}=\int\left(\int\left(E^{2}+\omega(\vec{p})^{2}\right)^{-1} \overline{\tilde{f}(\vec{p},-E)} \tilde{g}(\vec{p}, E) d \vec{p}\right) d E .
$$

Proposition VII.4 (Reflection Positivity). The form $\langle\cdot, \cdot\rangle_{\mathcal{H}}$ in (VII.11) is RP. The Hilbert space $\mathcal{H}=\left(\mathcal{E}_{+} / \mathcal{N}\right)^{-}$can be identified with $H_{-\frac{1}{2}}\left(R^{d-1}\right)$.

Remark. This Hilbert space $H_{-\frac{1}{2}}\left(R^{d-1}\right)$ enters naturally as the measure $(2 \omega(\vec{p}))^{-1} d \vec{p}$ is Lorentz invariant. It provides the standard space of wave functions $h(\vec{x})$ for relativistic quantum theory of a single, scalar particle. With a standard normalization, the inner product in this space can be expressed (in Fourier space) as

$$
\left\langle h_{1}, h_{2}\right\rangle_{H_{-\frac{1}{2}}}=\int \overline{\hat{h}_{1}(\vec{p})} \hat{h}_{2}(\vec{p}) \frac{d \vec{p}}{2 \omega(\vec{p})} .
$$

Proof. Observe that the partial Fourier transform in the time,

$$
\tilde{f}(\cdot, E)=\frac{1}{\sqrt{2 \pi}} \int e^{i E t} f(\cdot, t) d t
$$

of an element $f \in \mathcal{E}_{+}$extends to a holomorphic function in the upper-half $E$ plane. Likewise $\overline{\tilde{f}(\cdot,-E)}$ extends to a holomorphic function in the upper-half plane. One can evaluate the $E$ integral in (VII.11) using Cauchy's theorem as

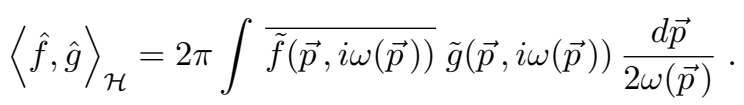

The inverse Fourier transform on $R^{d-1}$ of $\tilde{g}(\vec{p}, i \omega(\vec{p}))$ is the function

$$
G(\cdot)=\frac{1}{\sqrt{2 \pi}} \int_{0}^{\infty} e^{-t \omega} g(\cdot, t) d t .
$$

Inserting this representation in (VII.14) shows that

$$
\langle f, g\rangle_{\mathcal{H}}=\frac{1}{2}\langle F, G\rangle_{H_{-\frac{1}{2}}\left(R^{d-1}\right)}=\frac{1}{2} \int_{0}^{\infty} \int_{0}^{\infty}\left\langle f(\cdot, t), e^{-(t+s) \omega} g(\cdot, s)\right\rangle_{H_{-\frac{1}{2}}\left(R^{d-1}\right)} d t d s .
$$


In particular, the functions in $\mathcal{H}$ are that subset of $H_{-\frac{1}{2}}\left(R^{d-1}\right)$ mapped onto by the transform (VII.15). However, any element of the form $f=h \otimes \delta_{s}$ with $h \in H_{-\frac{1}{2}}\left(R^{d-1}\right)$ and $\delta_{s}$ the Dirac measure concentrated at time $s>0$ is an element of $\mathcal{E}_{+}$with $\hat{f}(p)=(2 \pi)^{-1 / 2} \hat{h}(\vec{p}) e^{i E s}$ and $F=$ $(2 \pi)^{-1 / 2} e^{-s \omega} h$. This is an element of $H_{-\frac{1}{2}}\left(R^{d-1}\right)$ that converges as $s \rightarrow 0+$ to $F=(2 \pi)^{-1 / 2} h$, so $\mathcal{H}$ contains all of $H_{-\frac{1}{2}}\left(R^{d-1}\right)$. Note that the kernel $\mathcal{N}$ of the map ^are those elements mapped by (VII.15) to zero. This is an infinite dimensional space; in the case $d=1$ it consists exactly of those functions $f(t)$ supported on $[0, \infty)$ and orthogonal to $e^{-t}$. The final remark about the embedding then follows by the Schwarz inequality on $\mathcal{E}$.

\section{VII.5 RP and Dirichlet-Neumann Monotonicity}

An interesting second interpretation of reflection positivity arises from the equivalence of RP with monotonicity of Green's operators for the Laplacian, corresponding to Dirichlet versus Neumann boundary data on the time-zero reflection plane, established in [19]. Notice that for Example 1, the Dirichlet Green's operator $C_{D}$ on the half-space $L^{2}\left(R_{+}^{d}\right)$ can be obtained by introducing a mirrorimage charge of opposite sign, reflected through the time-zero plane, namely

$$
C_{D}=\left(-\Delta_{D}+1\right)^{-1}=C-\Theta C .
$$

On $R_{+}^{d}$ this term $\Theta C$ satisfies the homogeneous equation $(-\Delta+1) \Theta C=0$, and it also assures that the resulting Green's function $C_{D}$ vanishes on the $t=0$ plane. Likewise, the Neumann Green's operator $C_{N}$, corresponding to the vanishing of the normal derivative to the $t=0$ plane has the image charge representation

$$
C_{N}=C+\Theta C .
$$

The statement of reflection positivity for $C$ on $R^{d}$ is the statement $0 \leq \Theta C \uparrow \mathcal{E}_{+}$. By subtracting the two representations (VII.17)-(VII.18), one sees that reflection positivity of the form (VII.11) is equivalent to the operator monotonicity of the Green's function in time-zero boundary conditions,

$$
C_{D} \leq C_{N} \uparrow L^{2}\left(R_{+}^{d}\right) .
$$

One can directly establish this monotonicity, yielding an alternative proof of reflection positivity that applies also to more general geometries than the one considered here [33, 34].

\section{VII.6 RP-2: The Fock Space of a Free, Scalar Quantum Field}

For the second example of RP, take $\mathcal{E}$ equal to the Schwartz space $\mathcal{S}_{\text {real }}^{\prime}\left(R^{d}\right)$ of real, tempered distributions on $R^{d}$. For this example choose $\mathcal{E}=L^{2}\left(d \mu_{C}\right)$, where $d \mu_{C}$ is the Gaussian probability measure on $\mathcal{S}_{\text {real }}^{\prime}\left(R^{d}\right)$ with mean zero and covariance $C=(-\Delta+1)^{-1}$. Such a measure is uniquely determined by its characteristic function (Fourier transform)

$$
S(f)=\int_{\mathcal{S}_{\text {real }}^{\prime}\left(R^{d}\right)} e^{i \Phi(f)} d \mu_{C}(\Phi)=e^{-\frac{1}{2}\langle f, f\rangle_{H_{-1}\left(R^{d}\right)}} .
$$

Here $f \in \mathcal{S}_{\text {real }}\left(R^{d}\right)$ is a function, and $\Phi(f)$ denotes the pairing between $\mathcal{S}_{\text {real }}^{\prime}$ and $\mathcal{S}_{\text {real }}$ which for functions $\Phi$ equals

$$
\Phi(f)=\int \Phi(x) f(x) d x
$$

The space of finite linear combinations of exponentials $\sum_{j} c_{j} e^{i \Phi\left(f_{i}\right)}$ is dense in $\mathcal{E}$. For two such functions $A, A^{\prime} \in \mathcal{E}$, one has

$$
\left\langle A, A^{\prime}\right\rangle_{\mathcal{E}}=\int_{\mathcal{S}^{\prime}\left(R^{d}\right)} \bar{A} A^{\prime} d \mu_{C}=\sum_{j, j^{\prime}} \overline{c_{j^{\prime}}^{\prime}} c_{j} S\left(f_{j}-f_{j^{\prime}}^{\prime}\right) .
$$


Likewise the subspace of finite linear combinations for which the support of $f_{j}$ is in the positive-time subspace $R_{+}^{d}$ is dense in $\mathcal{E}_{+}$. Similarly define $\mathcal{E}_{-}$. The time reflection $\Theta$ of Example 1 lifts to an action on $\mathcal{S}$ and on $\mathcal{S}^{\prime}$. Furthermore, $\Theta \mathcal{E}_{ \pm}=\mathcal{E}_{\mp}$. Define the sesqui-linear form $\langle\cdot, \cdot\rangle_{\mathcal{H}}$ on $\mathcal{E}_{+} \times \mathcal{E}_{+}$. For $A, A^{\prime} \in \mathcal{E}_{+}$, let

$$
\left\langle A, A^{\prime}\right\rangle_{\mathcal{H}}=\left\langle\Theta A, A^{\prime}\right\rangle_{\mathcal{E}}=\int \overline{\Theta A} A^{\prime} d \mu_{C}
$$

Proposition VII.5 (Gaussian RP). Let $d \mu_{C}$ be the Gaussian measure (VII.20) and $A \in \mathcal{E}_{+}$. Then $0 \leq\langle A, A\rangle_{\mathcal{H}}$. The Hilbert space $\mathcal{H}=\left(\mathcal{E}_{+} / \mathcal{N}\right)^{-}$equals the bosonic Fock space $\mathcal{F}$ over the one-particle space $H_{-\frac{1}{2}}\left(R^{d-1}\right)$ defined in $\S I V .2$.

Remark. In other words it is the symmetric tensor algebra over $H_{-\frac{1}{2}}$,

$$
\mathcal{F}=\oplus_{n=0}^{\infty} \mathcal{F}_{n}=C \oplus \oplus_{n=1}^{\infty} \underbrace{H_{-\frac{1}{2}} \otimes_{s} \cdots \otimes_{s} H_{-\frac{1}{2}}}_{n \text { times }}
$$

Proof. To establish reflection positivity, we reduce this example to Example 1. The fact that $d \mu$ is a probability measure requires that $S(f)$ be continuous, normalized $S(0)=1$, and positive definite. The latter property means that for any choice of $n$ functions $f_{1}, \ldots, f_{n} \in \mathcal{S}_{\text {real }}$, and complex constants $c_{1}, \ldots, c_{n}$, the element $A=\sum_{j=1}^{n} c_{j} e^{i \Phi\left(f_{j}\right)} \in \mathcal{E}_{+}$must yield a non-negative value of the form

$$
0<\langle A, A\rangle_{\mathcal{E}}=\int|A|^{2} d \mu_{C}=\sum_{j, j^{\prime}=1}^{n} \overline{d_{j^{\prime}}} d_{j} e^{\left\langle f_{j^{\prime}}, f_{j}\right\rangle_{H_{-1}}}, \quad \text { where } d_{j}=c_{j} S\left(f_{j}\right)
$$

In other words the matrix $e^{\left\langle f_{j^{\prime}}, f_{j}\right\rangle_{H_{-1}}}$ must be positive definite. This property is equivalent to the fact that $\left\langle f_{j^{\prime}}, f_{j}\right\rangle_{H_{-1}}$ is a positive-definite matrix, which in turn is equivalent to the fact that $C$ is a positive transformation on $L^{2}\left(R^{d}\right)$ with no null vector.

In a similar fashion, we obtain a condition equivalent to $\langle\cdot, \cdot\rangle_{\mathcal{H}}$ being reflection positivity. For every finite choice of real functions $f_{j}$ supported in $R_{+}^{d}$, and for all complex constants $c_{1}, \ldots, c_{n}$, one must have

$$
0 \leq\langle\hat{A}, \hat{A}\rangle_{\mathcal{H}}=\int \overline{\Theta A} A d \mu_{C}=\sum_{j, j^{\prime}=1}^{n} \overline{d_{j^{\prime}}} d_{j} e^{\left\langle f_{j^{\prime}}, \Theta f_{j}\right\rangle_{H_{-1}}}, \quad \text { where } d_{j}=c_{j} S\left(f_{j}\right)=c_{j} S\left(\Theta f_{j}\right)
$$

This is equivalent to the non-negativity of the matrix $e^{\left\langle f_{j^{\prime}}, \Theta f_{j}\right\rangle_{H_{-1}}}$, which is equivalent to the nonnegativity of $\left\langle f_{j^{\prime}}, \Theta f_{j}\right\rangle_{H_{-1}}$, which is equivalent to $0 \leq \Theta C\left\lceil L^{2}\left(R_{+}^{d}\right)\right.$. Thus we have reduced reflection positivity for Example 2 to the reflection positivity established in Example 1. For more details, see for example [20].

Now we identify $\mathcal{H}$. For real $f \in H_{-1}\left(R_{+}^{d}\right)$, define $n^{\text {th }}$-degree polynomials $P_{n}$ in the variable $\Phi(f)$ with leading order terms

$$
P_{n}(\Phi(f))=\Phi(f)^{n}+O\left(\Phi(f)^{n-2}\right)=(c / 2)^{n / 2} H_{n}\left((2 c)^{-1 / 2} \Phi(f)\right)
$$

where $c=c(f)=\int \Phi(f)^{2} d \mu_{C}(\Phi)=\|f\|_{H_{-1}}^{2}$, and where $H_{n}(\xi)$ denotes the standard Hermite polynomial in $\xi$. In particular, the generating function for these polynomials is

$$
F(z, f)=\sum_{n=0}^{\infty} \frac{(i z)^{n}}{n !} P_{n}(\Phi(f))=\exp \left(i z \Phi(f)+\frac{1}{2} z^{2}\|f\|_{H_{-1}\left(R^{d}\right)}^{2}\right)
$$


The polynomials $P_{n}(\Phi(f))$ are also called the normal ordered powers of $\Phi(f)$, and often one writes

$$
P_{n}(\Phi(f))=: \Phi(f)^{n}:
$$

From (VII.20) we infer

$$
\left\langle\widehat{F}\left(z^{\prime}, f^{\prime}\right), \widehat{F}(z, f)\right\rangle_{\mathcal{H}}=\left\langle F\left(z^{\prime}, f^{\prime}\right), \Theta F(z, f)\right\rangle_{L^{2}\left(d \mu_{C}\right)}=e^{z^{\prime} z\left\langle f^{\prime}, \Theta f\right\rangle_{H_{-1}}}
$$

It follows that the polynomials $\widehat{P}_{n}(\Phi(f))$ are orthogonal in $\mathcal{H}$ for different values of $n$, and that

$$
\left\langle\widehat{P}_{n}(\Phi(f)) \widehat{P}_{n^{\prime}}\left(\Phi\left(f^{\prime}\right)\right)\right\rangle_{\mathcal{H}}=\delta_{n n^{\prime}} n !\left\langle f, \Theta f^{\prime}\right\rangle_{H_{-1}\left(R^{d}\right)}^{n} .
$$

We have an important special case when $f=h \otimes \delta_{s}$, where $h \in H_{-\frac{1}{2}}$ and $\delta_{s}$ is the Dirac measure localized at time $0 \leq s$. Then from (VII.16) we remark that

$$
\left\langle f, \Theta f^{\prime}\right\rangle_{H_{-1}\left(R^{d}\right)}=\frac{1}{2}\left\langle h, e^{-\left|s-s^{\prime}\right| \omega} h^{\prime}\right\rangle_{H_{-\frac{1}{2}}\left(R^{d-1}\right)} .
$$

One can perform a similar calculation with $F(\vec{z}, \vec{h})=\prod_{j=1}^{N} F\left(z_{j}, h_{j} \otimes \delta_{0}\right)$, where $\left\{h_{j}\right\}$ are elements of a real orthogonal basis for $H_{-\frac{1}{2}}\left(R^{d-1}\right)$. Then for real $\vec{z}, \vec{z}^{\prime}$ and for $0 \leq t$, that

$$
\left\langle\widehat{F}\left(\vec{z}^{\prime}, \vec{h}^{\prime}\right), e^{-t H} \widehat{F}(\vec{z}, \vec{h})\right\rangle_{\mathcal{H}}=\exp \left(\sum_{j=1}^{N} \sum_{j^{\prime}=1}^{N^{\prime}} z_{j^{\prime}}^{\prime} z_{j}\left\langle h_{j^{\prime}}^{\prime}, e^{-t \omega} h_{j}\right\rangle_{H_{-\frac{1}{2}}\left(R^{d-1}\right)}\right) .
$$

Expanding the left side of (VII.28) in the case $t=0$ shows that the polynomials of the form

$$
\Omega_{\vec{n}}(\Phi)=\prod_{i} \frac{2^{n_{i} / 2}}{\sqrt{n !}} P_{n_{i}}\left(\Phi\left(h_{i} \otimes \delta\right)\right), \quad \text { with }|\vec{n}|=\sum_{i} n_{i}<\infty
$$

are an ortho-normal basis for $\mathcal{H}$, the tensor algebra over $H_{-\frac{1}{2}}\left(R^{d-1}\right)$. Those polynomials with $|\vec{n}|=n$ become a basis for the $n$-particle space; in fact there is an isomorphism between the vectors $\Omega_{\vec{n}} \in \mathcal{H}$ and the unit vectors $\otimes_{s i}\left(n_{i} !^{-1 / 2}\left(h_{i}^{\otimes_{s} n_{i}}\right)\right) \in \mathcal{F}_{n}$ of $\S I V .2$. It is in this sense that $\mathcal{H}=\oplus_{n} \mathcal{F}_{n}$.

By differentiating the definition (VII.28), one obtains the identity

$$
\Phi(f) F(z, f)=-i \frac{\partial F(z, f)}{\partial z}+i z\|f\|_{H_{-1}}^{2} F(z, f),
$$

and therefore the power series in $z$ yields the recursion relation

$$
\Phi(f) P_{n}(\Phi(f))=P_{n+1}(\Phi(f))+n\|f\|_{H_{-1}}^{2} P_{n-1}(\Phi(f)), \quad \text { for } n \geq 1 .
$$

In terms of the normalized functions $\Omega_{n}=2^{n / 2} n !^{-1 / 2} P_{n}(\Phi(h \otimes \delta))$, with $\|h\|_{H_{-\frac{1}{2}}}=1$, this becomes

$$
\Phi(h \otimes \delta) \Omega_{n}=\frac{1}{\sqrt{2}}\left(\sqrt{n+1} \Omega_{n+1}+\sqrt{n} \Omega_{n-1}\right), \quad \text { for } n \geq 1,
$$

and $\Phi(h \otimes \delta) \Omega_{0}=\frac{1}{\sqrt{2}} \Omega_{1}=h$. 


\section{VII.7 Osterwalder-Schrader Quantization for Operators}

Reflection positivity not only gives rise to $\mathcal{H}$, but it also gives a canonical procedure to map certain operators on $\mathcal{E}_{+}$to operators on $\mathcal{H}$. We call this aspect of RP by the name OS-quantization. Begin with a linear operator $T: \mathcal{E}_{+} \rightarrow \mathcal{E}_{+}$, and preserving the null space $\mathcal{N}$. The quantization $\hat{T}$ is the operator that $T$ induces on $\mathcal{H}$.

Definition VII.6 (OS-Quantization). If $T: \mathcal{E}_{+} \rightarrow \mathcal{E}_{+}$preserves $\mathcal{N}$, then the OsterwalderSchrader quantization map $T \mapsto \hat{T}$ to operators on $\mathcal{H}$ is given by ${ }^{2}$

$$
\hat{T} \hat{f}=\widehat{T f},
$$

or in terms of a commutative diagram,

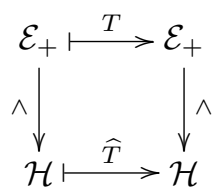

If $T$ has norm $\|T\|_{\mathcal{E}_{+}}<\infty$ on $\mathcal{E}_{+}$, then $\hat{T}$ is bounded on $\mathcal{H}$ with $\|\hat{T}\|_{\mathcal{H}} \leq\|T\|_{\mathcal{E}_{+}}$, and the closure of $\hat{T}$ has domain $\mathcal{H}$. In case the transformation $T$ does not map all of $\mathcal{E}_{+}$into $\mathcal{E}_{+}$, one may still be able to obtain a densely-defined quantization $\hat{T}$. One requires that $T$ be defined on a subset $\mathcal{D} \subset \mathcal{E}_{+}$, and that $T$ maps this domain into $\mathcal{E}_{+}$. While $\mathcal{D}$ may not be dense in $\mathcal{E}_{+}$, we require that $\widehat{\mathcal{D}} \subset \mathcal{H}$ is dense in $\mathcal{H}$. We call such a domain $\mathcal{D}$ a quantization domain. Sufficient conditions that ensure the existence of an unbounded quantization or the quantization of an unbounded $T$ can be found in $[32,33]$.

Although this quantization procedure is abstract, by some miracle it reproduces the correct Schrödinger-Heisenberg quantum theory in any non-relativistic quantum theory on $R^{N}$. And furthermore it also agrees not only with the quantization of free quantum fields, but also with the quantization of those interacting quantum fields with cutoffs preserving reflection positivity, as well as those for which cutoffs have been removed. This procedure also reproduces quantum theory with fermion fields, to higher-spin fields, and gauge fields, as long as the interaction satisfies reflection positivity. It also generalizes to some situations with symmetry different from Euclidean symmetry, such as the case of static time universes, see [33]. In the next sections we illustrate this quantization method for certain operators. These examples occur in reflection-positive inner products introduced in $\S$ VII.4 and $\S$ VII.6.

\section{VII.8 Euclidean Symmetry Yields Poincaré Symmetry}

The path from unitary Euclidean symmetry to unitary Poincaré symmetry takes two steps: quantization and analytic continuation. We describe this procedure now in the case of the representation arising for the spin-zero, mass-one particle and the corresponding representation for the scalar free field with mass one. We consider the quantization of various subgroups of the Euclidean group; this yields both bounded and unbounded semigroups, which analytically continue in the parameters of this group. This results in unitary Poincaré symmetry.

In the original constructions, one obtained the analytic continuation of the quantized representation as a special case of quantizing and analytically continuing all the expectations of Euclidean

\footnotetext{
${ }^{2}$ OS-quantization can be regarded as a generalization of Toeplitz quantization, in which case^ denotes the projection map (Bergmann kernel) from an $L^{2}$ space $\mathcal{E}_{+}$over a domain $\mathcal{D}$ in $C^{N}$ to a Hardy sub-space of functions holomorphic in $\mathcal{D}$, and $T_{f}$ denotes the operator of multiplication on the $L^{2}$ space by a bounded function $f$. In this case $\hat{T}_{f}$ is the Toeplitz quantization of $f$.
} 
fields to obtain quantum fields [46]. The analysis of the group representations without introducing fields can appeal to mathematicians, so we explain this point of view [46, 13, 37, 14].

An interesting application of this construction is for the time-translation semigroup $T(t)$ that acts on $\mathcal{E}_{+}$for $t \geq 0$. Through quantization and analytic continuation we obtain in this example the unitary, irreducible, spin-zero, mass-one representation $U(\Lambda, a)$ of the Poincaré group. The Euclidean representation $T(O, a)$ commutes with the operator $C$. Therefore it acts as a unitary group $T(O, a)$ on $H_{-1}$. In Example 2, the fact that $T(O, a)$ commutes with $C$ entails the invariance of the measure $d \mu \mathrm{m}$ and $S(T(O, a) f)=S(f)$ and $T(O, a)$. As a consequence, in Example 2, $T(O, a)$ lifts to a unitary transformation on $\mathcal{E}=L^{2}\left(\mathcal{S}^{\prime}\left(R^{d}\right), d \mu\right)$.

While we present here the scalar Examples 1 and 2, there is a corresponding construction for fermions [46], for gauge theories [47], and for other higher spin equations, and well as for supersymmetric settings $[44,45]$.

Proposition VII.7. Consider Example 1 of $\S$ VII.4 and Example 2 of $\S$ VII.6. The unitary representation of Euclidean symmetry $T(R, a)$ has an $O S$-quantization $\hat{T}(R, a)$ on $\mathcal{H}$, and it analytically continues to a unitary representation $U(\Lambda, a)$ of the Poincaré group.

Proof. We outline the construction and consider the quantization $\widehat{T}(O, a)$ of various generating subgroups of the Euclidean group $T(O, a)$, and how they yield (after analytic continuation in certain parameters) to a unitary representation of corresponding subgroups of a representation $U(\Lambda, a)$ of the Poincaré group on $\mathcal{H}$.

The unitary time-translation semi-group $T(t)=T(I,(\overrightarrow{0}, t)$, with $0 \leq t$ has the property $\Theta T(t)^{*} \Theta=T(t)$, so for $f \in \mathcal{E}_{+}$,

$$
\langle\hat{f}, \widehat{T}(t) \hat{f}\rangle_{\mathcal{H}}=\langle\Theta f, T(t) f\rangle_{\mathcal{E}}=\langle T(-t) \Theta f, f\rangle_{\mathcal{E}}=\langle\Theta T(t) f, f\rangle_{\mathcal{E}}=\langle\widehat{T}(t) \hat{f}, \hat{f}\rangle_{\mathcal{H}},
$$

and $\widehat{T}(t)$ is self-adjoint.

Example 1. In this case, we read off from (VII.16) that

$$
\widehat{T}(t)=e^{-t \omega}=e^{-t H}, \quad \text { for } 0 \leq t .
$$

In Fourier space, $\widehat{T}(t)$ then acts as multiplication by $e^{-t \omega(\vec{p})}$. In this case $\|\widehat{T}(t)\|_{\mathcal{H}}=e^{-t} \leq 1$, or $I \leq H$. As $H$ is positive, the contraction semigroup $\widehat{T}(t)$ has an analytic continuation in $t$ to a unitary subgroup of time translations of the Poincaré group as $U(t))=T(i t)$.

On the other hand, the unitary spatial translations $T(\vec{a})=T(I, \vec{a}, 0)$ commute with $\Theta$, so its quantization is also unitary. In this case, we read off from (VII.16) that in Fourier space,

$$
\widehat{T}(\vec{a})=e^{i \vec{a} \cdot \vec{p}}, \quad \text { or on } \mathcal{H}, \quad \widehat{T}(\vec{a})=e^{-\vec{a} \cdot \vec{\nabla}} .
$$

Thus defining the infinitesimal generator of spatial translations $\widehat{T}(\vec{a})$ on $\mathcal{H}$ as the momentum $\vec{P}$, one sees that $\vec{P}=-i \vec{\nabla}$ on $\mathcal{H}$ and that $\vec{P}$ acts as multiplication by the coordinate $\vec{p}$ in Fourier space. Define the unitary spatial translation subgroup of the Poincaré group as $U(\vec{a}))=e^{i \vec{a} \cdot \vec{P}}$.

Likewise, unitary spatial rotation subgroups of the Euclidean group $T_{i j}(\theta)$ (rotation by angle $\theta$ in the plane $\left(x_{i}, x_{j}\right)$, for $\left.1 \leq i<j \leq d-1\right)$, commute with $\Theta$ and quantize to unitary spatial translation subgroups of the Poincare group $U_{i} j(\theta)=\widehat{T}_{i j}(\theta)$. The infinitesimal generators of these subgroups are $L_{i j}=x_{i} P_{j}-x_{j} P_{i}$, where $x_{i}$ denotes multiplication by the corresponding coordinate on $H_{-\frac{1}{2}}\left(R^{d-1}\right)$, for $1 \leq i \leq d-1 .^{3}$

\footnotetext{
${ }^{3}$ One can also verify that this multiplication operator by $x_{j}$ on $H_{-1}\left(R^{d}\right)=\mathcal{E}$ has a quantization $\hat{x}_{j}$, for $1 \leq j \leq d-1$ which equals multiplication by the coordinate $x_{j}$ on $H_{-\frac{1}{2}}\left(R^{d-1}\right)=\mathcal{H}$. Furthermore, multiplication by the coordinate $t=x_{d}$ on $H_{-1}\left(R^{d}\right)=\mathcal{E}$ does not preserve the null space $\mathcal{N}$ of the quantization map, so $t$ does not have a quantization!
} 
Finally consider the unitary Euclidean rotations $T_{j}(\chi)$ by angle $\chi$ in the $\left(x_{j}, t\right)$ plane. This transformation does not map $\mathcal{E}_{+}$to $\mathcal{E}_{+}$, as it rotates some positive time points to points with negative time. However, $T_{j}(\chi)$ does map functions $\mathcal{D}$ supported in a wedge around the $t=0$ axis, of the opening angle $\frac{\pi}{2}-|\chi|$, into $\mathcal{E}_{+}$. For $|\chi|<\pi / 2$ the domain $\mathcal{D}$ is a quantization domain, and furthermore $T_{j}(\chi)$ preserves $\mathcal{N}$. The corresponding quantization

$$
\widehat{T}_{j}(\chi)=e^{\chi M_{j}},
$$

is unbounded. The transformation $M_{j}$ is self-adjoint on $\mathcal{H}=H_{-\frac{1}{2}}$ equal to the real line. The

unbounded family of operators $\widehat{T}_{j}$ has an analytic continuation to a unitary representation of the subgroup of the Lorentz group corresponding to boosts in the $\left(x_{j}, t\right)$ plane,

$$
U_{j}(\chi)=\widehat{T}_{j}(i \chi)=e^{i \chi M_{j}}, \quad \text { acting on } \mathcal{H}=H_{-\frac{1}{2}}\left(R^{d-1}\right) .
$$

The boost preserves the Minkowski form $t^{2}-x_{j}^{2}$ and acts as

$$
\left(x_{j}, t\right) \mapsto\left(x_{j} \cosh \chi+t_{j} \sinh \chi, x_{j} \sinh \chi+t_{j} \cosh \chi\right) .
$$

The infinitesimal generator of this action is $M_{j}=\omega x_{j}$, which is self-adjoint on $H_{-\frac{1}{2}}\left(R^{d-1}\right)$. From the $\left(d^{2}+d\right) / 2$ subgroups of the Poincaré group on Minkowski $d$-space analyzed here, one obtains the spin-zero, mass-one representation of the Poincaré group on $\mathcal{H}$ of Example 1.

Example 2. We have seen in Proposition VII.5 that the Hilbert space for Example 2 is obtained as a tensor algebra over the Hilbert space of Example 1. The representation $U$ acts on the $n$-particle space as an $n$-fold tensor product of the representation of Example 1.

\section{VII.9 The Simple Harmonic Oscillator}

The wave equation (IV.13) in this case is the simple harmonic oscillator equation with angular frequency 1 , namely

$$
\varphi_{t t}(t)+\varphi(t)=0
$$

The canonical commutation relations reduce to

$$
\left[\varphi_{t}(t), \varphi(t)\right]=-i, \quad \text { and } \quad[\varphi(t), \varphi(t)]=\left[\varphi_{t}(t), \varphi_{t}(t)\right]=0 .
$$

We now show how to recover this from the OS-quantization in the $d=1$ case of Example 2. In making this comparison, let us denote the coordinate $q=\varphi(0)$ by the usual symbol of non-relativistic quantum theory. One would like to identify the time evolution of this coordinate, $q(t)=U(t) q U(t)^{*}$, and the canonical momentum with $p=\varphi_{t}(0)$.

Starting with the quantization of $\mathcal{E}_{+}$, define $q=\widehat{\Phi}(0)$. In the 1-dimensional case, $h$ lies in a one-dimensional, one-particle space spanned by $\widehat{\Phi}(0)=\widehat{\Phi}(\delta)$. Then for $0 \leq t$,

$$
\left\langle\widehat{\Phi}(0), e^{-t H} \widehat{\Phi}(0)\right\rangle_{\mathcal{H}}=C(t)=\frac{1}{2} e^{-t},
$$

shows that $e^{-t H}=e^{-t}$ and $H=1$ on this space. Then (VII.33) simplifies (and has $N=N^{\prime}=1$ ) to

$$
\left\langle F(z), e^{-t H} F\left(z^{\prime}\right)\right\rangle_{\mathcal{H}}=e^{z z^{\prime} e^{-t}} .
$$

Expanding (VII.33) as a power series in $z$ and $z^{\prime}$ one concludes that

$$
\left\langle\Omega_{n}, e^{-H} \Omega_{m}\right\rangle_{\mathcal{H}}=e^{-n t} \delta_{n m} .
$$


In other words, the polynomials $\Omega_{n}(q) \in \mathcal{H}$ are ortho-normal eigenfunctions of $H$ with eigenvalue $n$. On functions of $q$, the inner product in the Hilbert space $\mathcal{H}$ reduces to the space

$$
\mathcal{H}=L^{2}(R ; d \nu(q)), \quad \text { where } d \nu(q)=\pi^{-1 / 2} e^{-q^{2}} d q .
$$

In this Schrödinger representation, the subspace $\mathcal{F}_{n}$ of the Fock representation is spanned by $H_{n}$. The action of the Hamiltonian $H$ in this representation is

$$
H=-\frac{1}{2} \frac{d^{2}}{d q^{2}}+q \frac{d}{d q} .
$$

Furthermore,

$$
\widehat{\Phi}(t)=e^{-t H} q e^{t H} .
$$

This operator analytically continues to

$$
q(t)=e^{i t H} q e^{-i t H}, \quad \text { or } q(i t)=\widehat{\Phi}(t) .
$$

Thus one sees that $p=-i d / d q+i q$. Hence the canonical commutation relations $[p, q]=-i$ and $[q, q]=[p, p]=0$ follow. Also $q(t)$ satisfies the oscillator equation,

$$
\ddot{q}(t)+q(t)=0 .
$$

The usual coordinate in Schrödinger quantum theory is $q=\hat{\Phi}(0)$, and the recursion relation (VII.37) can be written

$$
q \Omega_{n}(q)=\frac{1}{\sqrt{2}}\left(a^{*}+a\right) \Omega_{n},
$$

where $a^{*} \Omega_{n}=\sqrt{n+1} \Omega_{n+1}$ and $a \Omega_{n}=\sqrt{n} \Omega_{n-1}$.

\section{VII.10 Free Quantum Fields}

The general $d$-dimensional case of the oscillator construction above leads from the Gaussian measure $d \mu_{C}(\Phi)$ to the free quantum field defined in $\S I V .4$, in the form

$$
\widehat{\Phi}(\vec{x}, t)=\varphi(\vec{x}, i t)=e^{-t H} \varphi(\vec{x}) e^{t H} .
$$

The field $\varphi(\vec{x}, t)$ analytically continued to real time satisfies the Klein-Gordon equation (IV.13) and the the time-zero fields satisfy the canonical commutation relations (IV.15).

We have already concluded in Proposition VII.5 that the Hilbert space $\mathcal{H}$ arising from the quantization of $\mathcal{E}_{+}$in this case is the Fock space $\mathcal{F}$ over $H_{-\frac{1}{2}}\left(R^{d-1}\right)$. The invariant vacuum state is $\Omega=\widehat{1}$, and $H \Omega=0$. However unlike the previous section, in this case the Hermite polynomials are no longer eigenstates of $H$. In fact the spectrum of $H$ is continuous except for the eigenvalue 0 . The explicit identification of the quantization $\widehat{\Phi}(h \otimes \delta)$ of the Euclidean field with the time-zero free field $\varphi(h, 0)$ defined in $\S$ IV.2 arises from a calculation similar to the identification of the harmonic oscillator. It relies on the identity (VII.33).

\section{Non-Linear Fields}

The earlier discussion lays out a framework, but in itself does not solve any concrete existence problem for fields. In order to get to the non-linear examples, one must do a good deal of hard work to 
obtain a non-Gaussian, Euclidean invariant, reflection-positive measure $d \mu(\Phi)$, or its Fourier transform $S(f)=\int e^{i \Phi(f)} d \mu(\Phi)$. Then one can appeal to the general framework to give a corresponding quantum field.

In order to study the equation (V.1), one needs to obtain a measure like (VI.7), although the original one has no meaning because the factor

$$
\mathfrak{A}_{I}=\lambda\left\langle\Phi^{2}, \Phi^{2}\right\rangle_{L^{2}\left(R^{d}\right)}=\lambda \int_{R^{d}} \Phi(x)^{4} d x={ }_{\text {a.e. }} \infty .
$$

One must both regularize the quartic expression $\Phi(x)^{4}$ locally ("the ultra-violet problem"), and also truncate the integral over $R^{d}$ (the "infra-red problem"). Each of these regularizations require a modification to the action $\mathfrak{A}_{I}$ in order to remove it. As positivity is difficult to recover from non-positive approximations, in order to ensure reflection positivity of the limit, we require that the approximating measures be reflection positive.

The infra-red regularization will be performed by replacing $R^{d}$ by the torus $T^{d}$ with periods of length $\ell$. It is easy to verify that $C_{\ell}=(-\Delta+1)^{-1}$ on this torus is also reflection-positive with an appropriate, symmetric definition of time reflection on the torus. (The time reflection will interchange a positive-time and negative-time subspace.) In order to regularize the local singularity, replace $\Phi(\vec{x}, t)$ by $\Phi_{\kappa}(\vec{x}, t)$, where $\kappa$ denotes truncation of the Fourier series in the spatial variable $\vec{x}$. In this way we obtain an approximating measure,

$$
d \mu_{\kappa, \ell}(\Phi)=\frac{1}{Z_{\kappa, \ell}} e^{-\mathfrak{A}_{\kappa, \ell}(\Phi)} d \mu_{C_{\ell}}(\Phi)=\frac{1}{Z_{\kappa, \ell}} \exp \left(-\lambda \int_{T^{d}} \Phi_{\kappa}(x)^{4} d x\right) d \mu_{C_{\ell}}(\Phi),
$$

which is also reflection-positive. The plan is to take $\kappa \rightarrow \infty$ and then the period $\ell \rightarrow \infty$. The ultra-violet renormalization problem for $d>1$ forces this limit not to exist (unless $\lambda=0$ ). In fact from this point on one must specify $d$ in order to proceed. Up to now, only in the cases $d=2,3$ has an interesting limit been achieved.

In order to pass to the $\kappa \rightarrow \infty$ limit, one must modify the definition of $\lambda \Phi_{\kappa}(x)^{4}$. As a first step, one defines the normal-ordered powers : $\Phi_{\kappa}(x)^{n}:$. The name and notation of denoting these powers by colons comes from the physics literature. They are the scaled versions of the Hermite polynomials introduced earlier. Let

$$
c_{\kappa, \ell}=\left(\int \Phi_{\kappa}(x)^{2} d \mu_{C_{\ell}}(\Phi)\right) .
$$

Define the $n^{\text {th }}$ "normal-ordered powers" as

$$
: \Phi_{\kappa}(x)^{n}:=P_{n}\left(\Phi_{\kappa}(x)\right)=\left(c_{\kappa, \ell} / 2\right)^{n / 2} H_{n}\left(\left(2 c_{\kappa, \ell}\right)^{-1 / 2} \Phi_{\kappa}(x)\right),
$$

where $H_{n}$ is the same Hermite polynomial as in (VII.27). They are orthogonal with respect to the measure $d \mu(\Phi)$, and the leading degree term in : $\Phi_{\kappa}(x)^{n}:$ is $\Phi_{\kappa}(x)^{n}$. Also define $: \Phi(x)^{0}:=: 1:=1$. The first few normal ordered powers are

$$
\begin{array}{rlrl}
: \Phi_{\kappa}(x): & =\Phi_{\kappa}(x), & : \Phi_{\kappa}(x)^{2}:=\Phi_{\kappa}(x)^{2}-c_{\kappa, \ell}, \\
: \Phi_{\kappa}(x)^{3}: & =\Phi_{\kappa}(x)^{3}-3 c_{\kappa, \ell} \Phi_{\kappa}(x), & : \Phi_{\kappa}(x)^{4}:=\Phi_{\kappa}(x)^{4}-6 c_{\kappa, \ell} \Phi_{\kappa}(x)^{2}+3 c_{\kappa, \ell}^{2}, \\
& \ldots & &
\end{array}
$$

The constants in (VIII.5) proportional to powers of $c_{\kappa, \ell}$ are called renormalization constants. They diverge as $\kappa \rightarrow \infty$. It is a general feature of normal-ordered powers that for any $r$ functions $f_{1}, \ldots, f_{r} \in \mathcal{S}\left(R^{d}\right)$, the moments

$$
\int_{\mathcal{S}^{\prime}\left(R^{d}\right)}: \Phi_{\kappa}(x)^{n}: \Phi\left(f_{1}\right) \cdots \Phi\left(f_{r}\right) d \mu_{C_{\ell}}(\Phi)
$$


converge as $\kappa \rightarrow \infty$, and the resulting function is $C^{\infty}$ in the variable $x$. This defines the normalordered powers

$$
: \Phi(x)^{n}:=\lim _{\kappa \rightarrow \infty}: \Phi_{\kappa}(x)^{n}: \text {, }
$$

as densely defined, sesqui-linear forms on $L^{2}\left(d \mu_{C_{\ell}}\right) \times L^{2}\left(d \mu_{C_{\ell}}\right)$, with the domain $\mathcal{D} \times \mathcal{D}$. Here $\mathcal{D}$ is spanned by $\Phi\left(f_{1}\right) \cdots \Phi\left(f_{r}\right)$ for arbitrary $r$ and $f \in \mathcal{S}$. The resulting forms are $C^{\infty}$ in $x$.

Dimension $\mathbf{d}=2$. In the two dimensional case, it is sufficient to replace the $n^{\text {th }}$ power $\Phi(x)^{n}$ in the action density by the $n^{\text {th }}$ normal-ordered power,

$$
\Phi_{\kappa}(x)^{n} \mapsto: \Phi_{\kappa}(x)^{n}: \text {. }
$$

For even $n$, and arbitrary real $\alpha_{j}$, consider the polynomial actions

$$
\mathfrak{A}_{I}=V_{n}+\sum_{j=1}^{n-1} \alpha_{j} V_{j},
$$

and the similar action $\mathfrak{A}_{I, \kappa}$. We have already remarked that in a weak sense,

$$
\mathfrak{A}_{I}=\lim _{\kappa \rightarrow \infty} \mathfrak{A}_{I, \kappa} ;
$$

exists. However in dimension $d=2$ it is also the case that

$$
e^{-\mathfrak{A}_{I, \kappa}} \rightarrow e^{-\mathfrak{A}_{I}} \in L^{p}\left(d \mu_{C_{\ell}}(\Phi)\right), \quad \text { for all } 0<p<\infty .
$$

In particular the finite-volume $\Phi^{4}$ measure exists and equals

$$
d \mu_{\ell}(\Phi)=\frac{1}{Z_{\ell}} e^{-\lambda V_{4}} d \mu_{C_{\ell}}(\Phi) .
$$

Nelson had the first result pertaining to $V_{4}$ [40]; general proofs and extensive references can be found in $[20,51]$.

The proof of the existence of the infinite volume limit $\ell \rightarrow \infty$ is more complicated. Two approaches exist, one based on expansions called cluster expansions [20, 22]. This method is valid for $0 \leq \lambda \ll 1$ and also for $\lambda \gg 1$. The second method is based on general inequalities (correlation inequalities). It is valid for all $0<\lambda$ but does not yield information on the spectrum of $H$.

All the results follow from detailed a priori estimates, many of which one can establish within the Euclidean framework. But these estimates ensure estimates in the framework of quantum theory. Two of the most fundamental estimates are posed in terms of the Hamiltonian $H$, obtained as a perturbation of the free Hamiltonian. After renormalization one needs to establish that $H$ is a well-defined, positive operator

$$
0 \leq H,
$$

where we adjust the infimum of the spectrum of $H$ to be 0 . Secondly one needs to show that it is regular when perturbed locally by the time-zero field, and the inequality

$$
\pm \varphi(h) \leq\|h\|(H+I),
$$

where $\|h\|$ denotes a Schwartz-space norm of $h$. These fundamental estimates must be uniform in any cutoff approximations, and yield the required regularity of the quantum fields.

We summarize some more known results in the following two theorems; see [20] for further discussion and for references to the original proofs. 
Theorem VIII.1 (Glimm-Jaffe-Spencer, Guerra-Rosen-Simon, others). One $\lambda \varphi^{4}$ quantum field theory exists for each $\lambda$ with $0 \leq \lambda \ll 1$ sufficiently small. It satisfies the Osterwalder-Schrader axioms for Euclidean theory, the Wightman axioms for vacuum expectation values, the Haag-Ruelle axioms for scattering axioms, and the Haag-Kastler axioms of local quantum theory. The scattering matrix elements agree with the usual physics perturbation theory as an asymptotic expansion.

Theorem VIII.2 (Glimm-Jaffe-Spencer). Two $\lambda \varphi^{4}$ quantum field theories exist for each $\lambda$ with $1 \ll \lambda$ sufficiently large. This non-uniqueness arises from broken $\varphi \rightarrow-\varphi$ symmetry in the vacuum. Each theory satisfies the Osterwalder-Schrader axioms for Euclidean theory, the Wightman axioms for vacuum expectation values, and the Haag-Kastler axioms of local quantum theory.

The phenomenon of symmetry breaking is extremely important, and it has been elaborated in many directions. The theory of phase transitions in quantum field theory corresponds to the existence of multiple solutions for a given equation-i.e. a set of parameters in the action. In terms of the measure $d \mu(\Phi)$, symmetry breaking is absent when the measure is ergodic. Furthermore clustering is the effect of the mixing property of each ergodic component. Each different pure state in $\mathcal{H}$ (or ergodic component of the measure $d \mu$ ) is called a phase.

An extensive theory has been developed, and it parallels in part related results in statistical physics. But it impacts only peripherally on our main theme, so we mention here only a couple of points. In dimension $d=2$ one has examples with $n$ phases arising from polynomial interactions with $n$ global minima. In certain instances, Imbrie showed that perturbations of such a theory have a phase diagram which is topologically equivalent to the neighborhood of the corner of a hyper-cube: at the corner one finds $n$ coexisting phases; along lines coming from the corner one has $n-1$ phases; surfaces with $n-2$ coexisting phases, etc. In addition, multi-component fields lead to the analysis of continuous symmetry. Field theory methods have been used (especially by Fröhlich and Spencer) to analyze such problems in statistical physics. See [20] for some further references.

Dimension $\mathbf{d}=3$. The situation in dimension three is dramatically more complicated. One might at first think that the substitution (VIII.8) would also provide the renormalization of the threedimensional problem. The reason that one can easily be misled arises from the convergence (VIII.7), which is independent of dimension. This yields convergence of the action

$$
\mathfrak{A}_{I, \kappa}=\int_{T^{3}}: \Phi_{\kappa}(x)^{4}: d x
$$

to a limiting action

$$
\mathfrak{A}_{I}=\lim _{\kappa \rightarrow \infty} \mathfrak{A}_{I, \kappa},
$$

which is a sesqui-linear form on the the Fock space, with the domain of functions with a finite number of particles and Schwartz-space wave functions.

But such an action does not necessarily give rise to a field theory; one needs to be able to integrate powers of the action. In three dimensions the function $\mathfrak{A}_{I}^{2}$ is not integrable with respect to $d \mu_{C_{\ell}}(\Phi)$. For this reason, physicists regard the normal-ordering contribution to renormalization as a trivial renormalization!

In three dimensions, a more subtle change in the action is necessary for a non-linear equation. This change has only been justified for the $\lambda \varphi^{4}$ equation. In the Euclidean theory one chooses also a quadratic and a constant term. Thus in place of the substitution (VIII.8) in the action, in dimension $d=3$ one makes the substitution,

$$
\lambda \Phi_{\kappa}(x)^{4} \mapsto \mathfrak{A}_{\kappa}(x)=\lambda: \Phi_{\kappa}(x)^{4}:-\alpha_{\kappa} \lambda^{2}: \Phi_{\kappa}(x)^{2}:+\lambda^{2} \beta_{\kappa}-\lambda^{3} \gamma_{\kappa} .
$$


Then one studies

$$
\mathfrak{A}_{\kappa}=\int_{T^{3}} \mathfrak{A}_{\kappa}(x) d x, \quad \text { and } \quad d \mu_{\kappa, \ell}=\frac{1}{Z_{\kappa, \ell}} e^{-\mathfrak{A}_{\kappa}} d \mu_{C}(\Phi) .
$$

This is a very unusual-looking expression! First notice that unlike the logarithmic divergence in the $d=2$ case, here

$$
c_{\kappa} \sim \kappa,
$$

so the normal-ordering : $\Phi_{\kappa}(x)^{4}$ : is more singular. It has an order $\kappa$ quadratic term in $\Phi_{\kappa}$ and an order $\kappa^{2}$ constant term. The behavior of $c_{\kappa}$ is a consequence of the $\left|x-x^{\prime}\right|^{-1}$ singularity on the diagonal of the Green's function $C\left(x-x^{\prime}\right)$. Furthermore the renormalization constants $\alpha_{\kappa}, \beta_{\kappa}, \gamma_{\kappa}$ all diverge as $\kappa \rightarrow \infty$. The exact rate of divergence is not important, but the structure of the renormalization constants is. These constants are chosen to be proportional to the following integrals that arise in perturbation theory, so the rates of divergence are governed by the singularity on the diagonal of the Green's function $C(x-y)$. More precisely, there are numerical constants $\alpha, \beta, \gamma$, such that

$$
\alpha_{\kappa}=\alpha \int_{T^{3}} C_{\kappa}(x)^{3} d x \sim \ln \kappa, \quad \beta_{\kappa}=\beta \int_{T^{3}} C_{\kappa}(x)^{4} d x \sim \kappa ;
$$

and

$$
\gamma_{\kappa}=\gamma \int_{T^{3} \times T^{3}} C_{\kappa}(x)^{2} C_{\kappa}(x-y)^{2} C_{\kappa}(y)^{2} d x d y \sim \ln \kappa .
$$

After introducing these renormalization counter-terms into the action, one must prove that the resulting integrals are convergent, and that $e^{-\mathfrak{A}_{\kappa}}$ is $d \mu_{C}(\Phi)$-integrable, uniformly in $\kappa$. Furthermore one needs to exhibit convergence of the Fourier transform $S(f)$ of the measure as $\kappa \rightarrow \infty$. Then one can deal with the infinite-volume limit $\ell \rightarrow \infty$.

The first breakthrough on this problem came in one of the most complicated papers in the constructive quantum field theory literature [18], which proved that for any $0 \leq \lambda$, the density $e^{-\mathfrak{A}_{\kappa}}$ is integrable in $d \mu_{C}(\Phi)$, uniformly in $\kappa$, in a fixed, finite space-time volume. As a byproduct this work also proved the stability (boundedness from below) of a Hamiltonian for the finite-volume $\lambda \varphi^{4}$ equation in $d=3$ dimensions. Written almost thirty five years ago, the paper [18] has yet to be fully digested, and should be reinvestigated from a more modern perspective.

Feldman and Osterwalder [7] combined this result (in the case $0 \leq \lambda \ll 1$ ) with the cluster expansion method, to prove the existence of an infinite-volume theory satisfying the OsterwalderSchrader axioms. One can summarize part of these results in a theorem:

Theorem VIII.3 (Glimm and Jaffe, Feldman and Osterwalder). With $0 \leq \lambda \ll 1$, one has a unique Euclidean-invariant measure $\lambda \Phi^{4}$ on $\mathcal{S}^{\prime}\left(R^{3}\right)$. This measure is obtained as the limit of the Fourier transforms of the approximating measures (VIII.18) as $\kappa \rightarrow \infty$ and $\ell \rightarrow \infty$. The limiting measure satisfies the Osterwalder-Schrader axioms (including exponential clustering). Thus it yields a relativistic $\lambda \varphi^{4}$ quantum field theory satisfying the Wightman axioms with a unique vacuum and with a non-zero mass gap.

The methods in the proof of the stability bound fascinated George Mackey. They were based on the idea that one must analyze the integral over $d \mu(\Phi)$ in different regions of "phase space" of the field $\Phi(x)$, namely with approximate simultaneous localization both in $x$-space and the corresponding Fourier space variable $k$. One first performs the integral over degrees of freedom associated with sufficiently small $x$-size, namely $O\left(\kappa^{-2}\right)$, so that the renormalizations become finite. But one must simultaneously analyze large $k$-size, so the size of a phase cell (the product of the $x$-size and the $k$-size) is larger than of order $O(1)$. One then must show that the effects of different phase cells is approximately independent. After treating this scale, one proceeds to analyze a larger length scale 
of increased spatial size by the factor $L^{3}$, and corresponding decreased momentum size by the factor $L^{-3}$, etc. One proceeds inductively, until one has integrated all the degrees of freedom. In this fashion one proves that $e^{-\mathfrak{A}_{\kappa}}$ is integrable, and one also obtains a bound for the integral that is uniform in $\kappa$.

The idea of this proof has many features in common with Wilson's renormalization group theory that he developed during the same period of time [61]. One major difference is that the phase cell analysis led to an inductive proof, while Wilson's approach gives an iterative procedure - each successive step in his formulation has the same structure. An iterative method has the great advantage of simplicity and conceptual clarity, while an inductive method may be more robust.

In any case, a great deal of interesting work has gone into a new proof of the existence of the $\lambda \Phi^{4}$ measure using the renormalization group ideas, see especially $[6,4,5,39]$. However, while the local stability bound (only in the case $0 \leq \lambda \ll 1$ ) has been established by these methods, the major end results: convergence of the infinite volume theory, the existence of a theory satisfying the Wightman axioms, and the existence of a uniform mass gap have not been proved in this way. Thus it is very fruitful to study this question. Not only should one give a transparent proof of the dimension $d=3$ construction, but as explained to me by Gelfand [16], one should make it sufficiently attractive that probabilists will take cognizance of the existence of a wonderful new mathematical object: a one-parameter family of non-Gaussian, Euclidean-invariant, reflection-positive measures $d \mu_{\lambda}(\Phi)$ on $\mathcal{S}^{\prime}\left(R^{3}\right)$, indexed by the coupling constant $0<\lambda \ll 1$.

\section{Four Dimensions}

We have already mentioned that one does not know whether there is a logical (mathematical) theory behind the striking comparison between calculational rules and experiment in quantum electrodynamics. Based solely on its accuracy, one is forced to believe that quantum electrodynamics must ultimately rest on a mathematical foundation. The history of physics supports this assumption, as has been explained eloquently by Eugene Wigner in his famous essay on the rôle of mathematics in the natural sciences [60]. Yet here we meet a real anomaly.

If one continues the arguments that were successful in dimension $d=2,3$, then one would begin by noticing that for $d=4$, the normal ordering constant $c_{\kappa} \sim \kappa^{2}$. Thus even this elementary sort of renormalization will yield some (constant) divergence of order $\kappa^{4}$. Thus the phase-cell localization method apparently requires localization in phase volumes that are on the borderline of the $\mathrm{O}(1)$ limit imposed by Fourier analysis. Without some leeway in estimation, one can anticipate difficulty.

In fact, physicists have discovered an effect called asymptotic freedom that is special to quantum theory $[25,48]$. This effect distinguishes good non-linearities (asymptotically-free ones) from others that are bad (not asymptotically free). Asymptotic freedom goes one step beyond dimensional analysis by incorporating the high-energy behavior of perturbative corrections to the behavior of the scaling properties of action action $\mathfrak{A}_{I}$ compared that those of $\mathfrak{A}_{0}$. It provides a test that is especially useful in the borderline cases where the scaling behavior of $\mathfrak{A}_{I}$ is the same as the scaling behavior of $\mathfrak{A}_{0}$. These are also the cases for which phase-cell localization method is borderline.

Unfortunately neither quantum electrodynamics nor $\lambda \varphi^{4}$ interactions in four dimensions are asymptotically free. It is for this reason that today most physicists believe neither quantum electrodynamics nor the $\lambda \varphi^{4}$ equation in four dimensions have any solution with non-zero coupling constant. In fact, for the $\lambda \varphi^{4}$ theory one can prove under reasonable assumptions that in dimension $d \geq 5$ there is no non-linear solution $[12,1]$, but this proof only relies on the scaling dimension and not on the more subtle non-asymptotic freedom property. In the borderline case of four dimensions things have also not been resolved completely, although most indications point to the interaction being too singular to yield a non-linear theory.

One expects that for those interactions that are "asymptotically free," the renormalization 
transformations of renormalization group theory will flow toward a solution. We do not presently know if this picture can be fully turned into mathematics. But asymptotic freedom indicates that Yang-Mills theories for symmetry groups $S U(n)$ for $n \geq 2$ (the generalization of Maxwell theory to the case of Lie-algebra valued fields potentials) can have a field theory solution. Preliminary work on the finite-volume Yang-Mills theory by Balaban [2] suggests that the control of the ultra-violet problem using asymptotic freedom could well be brought fully into the arena of mathematics.

\section{IX.1 For the Future}

There is a related question which appears at present as a stumbling block to passing to the infinitevolume limit for the asymptotically-free Yang-Mills equations. This is the question of uniform clustering, or the existence of a uniform gap in the mass spectrum. As a technical matter, the robust methods alluded to in two and three dimensions obtain infinite volume limits (in the known examples) by proving exponential decay of correlations at large distances with a rate that is uniform in the volume. They use this property in order to prove the existence of the infinite volume limit: one studies the expectation of observables $A$ localized in a fixed cube $X$. One adds to the interaction a piece $\mathfrak{A}(\Delta)$ localized in a unit cube $\Delta$ far from $X$. Using exponential decay of correlations between $A$ and $\mathfrak{A}(\Delta)$, one shows convergence of the sum over $\Delta$ 's covering the complement of $X$.

The problem with the Yang-Mills theory is that when one sets the Yang-Mills coupling equal to zero, the equations reduce to Maxwell theory. And in Maxwell theory the particles are photons, which have zero mass. These do not yield correlations with exponential decay. Therefore, if one wants to establish that the particles have a positive mass for non-zero coupling (and that the Hamiltonian has a mass gap), then one needs to understand the basis in physics for the generation of the mass gap. One also needs to understand how to implement this idea in mathematics, so one can prove the existence of a uniform gap in a sequence of approximating theories.

In the physics literature, a mass gap for gauge theories appears natural in a finite volume, but how to show that the gap is uniform in the volume is unclear. While there are many proposals, the mechanism is not fundamentally understood. The answer to this question appears related to the famous problem of confinement: in particle physics quarks are believed to be the fundamental building blocks of particles in nature; yet quarks have never been observed experimentally! Thus one surmises that quarks must be bound together by a strong, confining force, one which increases with distance.

Physicists have worked for years in trying to understand a fundamental mechanism for this effect. But none of these potential explanations has been established from first principles, nor to all physicists' satisfaction. The problem is so fundamental that many physicists assume that the answer is "obvious;" yet no one really understands it! Thus an essential new feature necessary to solve the mathematical problem of obtaining a theory in (infinite-volume) Minkowski space appears to be a clear physical understanding of confinement. But by finding a solution, one can make a major contribution both to physical insight into these equations, as well as to mathematics.

Short of discovering some exactly-soluble example, the non-abelian Yang-Mills equations appear to provide the best hope for finding a non-linear quantum field in four dimensions. One can be optimistic that someday one will have a positive answer to this mathematical problem, yielding an example of the Wightman axioms with interaction. One can read more about this in [35].

\section{References}

[1] Michael Aizenman, Geometric Analysis of $\varphi^{4}$ Fields and Ising Models. Parts I and II, Comm. Math. Phys. 86 (1982), 1-48. 
[2] Tadeusz Balaban, Renormalization group approach to lattice gauge field theories. I. Generation of effective actions in a small field approximation and a coupling constant renormalization in 4D, Commun. Math. Phys. 109 (1987), 249-301.

[3] Hans J. Borchers, On the Algebra of Field Observables, Nuovo Cimento 24 (1962), 214-236.

[4] David Brydges, Jonathan Dimock, Thomas Hurd, Weak perturbations of Gaussian measures, Applications of the renormalization group, in CRM Proceedings \& Lecture notes, Mathematical quantum theory I: Field theory and many body theory, pages 1-28, 171-190, 1994. , The short distance behaviour of $\phi_{3}^{4}$, Comm. Math. Phys., 172 (1995), 143-186.

[6] David Brydges, Hong-Tzer Yau, Grad $\varphi$ perturbations of massless Gaussian fields, Comm. Math. Phys., 129 (1990), 351-392.

[7] Joel Feldman and Konrad Osterwalder, The Wightman axioms and the mass gap for weakly coupled $\phi_{3}^{4}$ quantum field theories, Ann. Physics, 97 (1976), 80-135.

[8] Richard Feynman, see the discussion on pages 226-228, following the lecture by Arthur Wightman in Proceedings of the International Conference on Particles and Fields, Rochester, N. Y., 1967, edited by C. R. Hagen, G. Guralnik, and V. S. Mathur, Interscience Publishers, New York 1967.

[9] , QED: the Strange Theory of Light and Matter, Princeton University Press, Princeton, 1985.

[10] Kurt O. Friedrichs, Mathematical Aspects of the Quantum Theory of Fields, Interscience, New York, 1953.

[11] Jürg Fröhlich, Verification of Axioms for Euclidean and Relativistic Fields and Haag's Theorem in a Class of $P(\varphi)_{2}$-Models, Ann. Inst. Henri Poincaré, XXI (1974), 271-317.

[12] _ On the Triviality of $\lambda \varphi_{d}^{4}$ Theories and the Approach to the Critical Point in $d \geq 4$ Dimensions, Nuclear Physics, B200 [FS4] (1982), 281-296.

[13] Unbounded, Symmetric Semigroups on a Separable Hilbert Space are Essentially Self Adjoint, Advances in Applied Mathematics, 1 (1980), 237-256.

[14] Jürg Fröhlich, Konrad Osterwalder, and Erhard Seiler, On Virtual Representations of Symmetric Spaces and Their Analytic Continuation, Ann. Math. 118 (1983), 461-489.

[15] G. Gabrielse, D. Hanneke, T. Kinoshita, M. Nio, and B. Odom, New Determination of the Fine Structure Constant from the Electron $g$ Value and QED, Phys. Rev. Lett. 97 (2006), 030802, and Erratum, (2007).

[16] Israel Gelfand, private communication.

[17] James Glimm and Arthur Jaffe, A $\lambda \phi^{4}$ Quantum Field Theory without Cut-offs. I, Phys. Rev., 176 (1968), 1945-1961 and reprinted in [21].

[18] _ Positivity of the $\phi_{3}^{4}$ Hamiltonian, Fortschritte der Physik, 21 (1973), 327-376 and reprinted in [21].

[19] _ A Note on Reflection Positivity, Lett. Math. Phys., 3 (1979), 377-378.

[20] , Quantum Physics: a Functional Integral Point of View, Springer-Verlag, New York, 1981, 1987. 
[21] _ Collected Papers, Vol. II: Constructive Quantum Field Theory, Selected Papers, with J. Glimm, 533 pages, Birkhäuser Boston (1985).

[22] James Glimm, Arthur Jaffe, and Thomas Spencer, The Wightman Axioms and Particle Structure in the $P(\phi)_{2}$ Quantum Field Model, Ann. of Math., 100 (1974), 585-632.

[23] _ Phase Transitions for $\phi_{2}^{4}$ Quantum Fields, Commun. Math. Phys., 45 (1975), 203-216.

[24] _ A Convergent Expansion about Mean Field Theory, Part I. The Expansion, Ann. Phys. 101 (1976), 610-630, and Part II. Convergence of the Expansion, Ann. Phys. 101 (1976), 631-669.

[25] D. J. Gross and F. Wilczek, Ultraviolet behavior of non-abelian gauge theories, Phys. Rev. Lett. 30 (1973), 1343-1346.

[26] Francesco Guerra, Uniqueness of the vacuum energy density and van Hove phenomenon in the infinite volume limit for two-dimensional self-coupled Bose fields, Phys. Rev. Lett. 28 (1972), $1213-1215$

[27] Francesco Guerra, Lon Rosen, and Barry Simon, The $\mathcal{P}(\varphi)_{2}$ Euclidean quantum field theory as classical statistical mechanics, Ann. Math. 101 (1975), 111-259.

[28] Rudolf Haag, Local Quantum Physics, Springer-Verlag, Berlin, 1996.

[29] Arthur Jaffe, Dynamics of a Cut-off $\lambda \phi^{4}$ Field Theory, Princeton University Thesis (1965). A summary is published in Existence Theorems for a Cut-off $\lambda \phi^{4}$ Field Theory, in Mathematical Theory of Elementary Particles, R. Goodman and I. Segal (eds.), MIT Press, Cambridge, 1966.

[30] Constructive quantum field theory, in Mathematical Physics 2000, edited by T. Kibble, 111-127, Imp. Coll. Press, London, 2000.

[31] _ Lunch with George, Notices of the American Mathematical Society 54 (2007), $833-836$.

[32] _ Introduction to Quantum Field Theory, Lecture Notes, partially available at http://www.arthurjaffe.com/Assets/pdf/IntroQFT.pdf

[33] Arthur Jaffe and Gordon Ritter, Quantum Field Theory on Curved Backgrounds, II. Spacetime Symmetries, Comm. Math. Phys., to appear.

[34] _ Reflection Positivity and Monotonicity, preprint.

[35] Arthur Jaffe and Edward Witten, Quantum Yang-Mills Theory, in Millennium Prize Problems, American Mathematical Society, Providence, RI, 2006.

[36] Res Jost, The General Theory of Quantized Fields, American Mathematical Society, Providence, 1965.

[37] Abel Klein and Lawrence Landau, Construction of a Unique Selfadjoint Generator for a Symmetric Local Semigroup, Jour. Funct. Anal., 44 (1981), 121-137, and From the Euclidean Group to the Poincaré Group via Osterwalder-Schrader Positivity, Commun. Math. Phys., 87 (1983), 469-484. 
[38] Ettore Majorana, Teoria relativistica di particelle con momento intrinseco arbitrario, Nuovo Cimento 9 (1932), 335-344.

[39] David Moser, Renormalization of $\varphi_{3}^{4}$ Quantum Field Theory, Diploma Thesis, E.T.H. Zürich, September 2006.

[40] Edward Nelson, A quadratic interaction in two dimensions, in Conference on the Mathematical Theory of Elementary Particles, R. Goodman and I. Segal, Eds., MIT Press, Cambridge, 1966.

[41] _ The Free Markov Field, J. Funct. Anal. 12 (1973), 211-217.

[42] The Construction of Quantum Fields from Markov Fields, J. Funct. Anal. 12 (1973), 97-112.

[43] B. Odom, D. Hanneke, B. D'Urso, and G. Gabrielse, New Measurement of the Electron Magnetic Moment Using a One-Electron Quantum Cyclotron, Phys. Rev. Lett. 97 (2006), 030801 .

[44] Konrad Osterwalder, Supersymmetric Quantum Field Theory, in Constructive Physics (Palaiseau, 1994), Lecture Notes in Phys., 446, Edited by V. Rivasseau, pages 117-130, Springer, Berlin, 1995.

[45] _ Constructing Super-symmetric Quantum Field Theory Models, In Advances in Dynamical Systems and Quantum Physics (Capri, 1993), pages 209-224, World Sci. Publ., River Edge, NJ, 1995.

[46] Konrad Osterwalder and Robert Schrader, Axioms for Euclidean Green's functions, Commun. Math. Phys. 31 (1973), 83-112, and Commun. Math. Phys. 42 (1975), 281-305.

[47] Konrad Osterwalder and Erhard Seiler, Gauge Theories on a Lattice, Ann. Phys., 110 (1978), $440-471$.

[48] H. D. Politzer, Reliable perturbative results for strong interactions? Phys. Rev. Lett. 30 (1973), 1346-1349.

[49] Julian Schwinger, On the Euclidean structure of relativistic field theory, Proc. Natl. Acad. Sci. U.S.A. 44 (1958), 956-965.

[50] _ Four-dimensional Euclidean Formulation of Quantum Field Theory, in Proceedings of the 1958 International Conference on High Energy Physics, CERN, Geneva, edited by B. Ferretti, CERN Scientific Information Service, Geneva, 1958, pages 134-140.

[51] Barry Simon, The $P(\phi)_{2}$ Euclidean (quantum) field theory, Princeton University Press, Princeton, N.J., 1974.

[52] Irving E. Segal, Mathematical Problems of Relativistic Physics, with an appendix by George W. Mackey, American Mathematical Society, Providence, 1963.

[53] Raymond Streater and Arthur Wightman, PCT, Spin and Statistics and all That, W. A. Benjamin, New York, 1964.

[54] Kurt Symanzik, A Modified Model of Euclidean Quantum Field Theory, Courant Institute of Mathematical Sciences, New York University Report IMM-NYU-327 comprising 132 pages, preprint June 1964, unpublished. 
[55] _ Euclidean Quantum Field Theory, in Local Quantum Theory, pp. 152-226, R. Jost, Editor, Academic Press, New York, 1969.

[56] Arthur S. Wightman, Quantum Fields in Terms of Vacuum Expectation Values, Phys. Rev. 101 (1956), 860-866.

[57] Arthur Wightman and Lars Gårding, Fields as Operator-Valued Distributions in Relativistic Quantum Field Theory, Arkiv Fysik 28 (1965), 128-184.

[58] David V. Widder, Necessary and sufficient conditions for the representation of a function by a doubly infinite laplace integral, Bull. Amer. Math. Soc., 40 (1934), 321-326.

[59] Eugene P. Wigner, On Unitary Representations of the Inhomogeneous Lorentz Group, Ann. Math. 40 (1939), 149-204.

[60] _ The Unreasonable Effectiveness of Mathematics in the Natural Sciences, Comm. Pure Appl. Math. 13 (1960) 1-14. Reprinted in Symmetries and Reflections: Scientific Essays of Eugene P. Wigner, pages 222-237, M.I.T. Press, Cambridge, 1970.

[61] Kenneth G. Wilson and John Kogut, The Renormalization Group and the $\epsilon$-Expansion, Physics Reports 12C (1974), 76-200. 